Noname manuscript No.

(will be inserted by the editor)

\title{
Photoabsorption in Sodium Clusters: First Principles Configuration Interaction Calculations
}

\author{
Pradip Kumar Priya, Deepak Kumar Rai, and \\ Alok Shukla
}

the date of receipt and acceptance should be inserted later

\begin{abstract}
We present systematic and comprehensive correlated-electron calculations of the linear photoabsorption spectra of small neutral closed- and open-shell sodium clusters $\left(\mathrm{Na}_{n}, \mathrm{n}=2-6\right)$, as well as closed-shell cation clusters $\left(\mathrm{Na}_{n}{ }^{+}, \mathrm{n}=3,5\right)$. We have employed the configuration interaction (CI) methodology at the full CI (FCI) and quadruple CI (QCI) levels to compute the ground, and the low-lying excited states of the clusters. For most clusters, besides the minimum energy structures, we also consider their energetically close isomers. The photoabsorption spectra were computed under the electric-dipole approximation, employing the dipole-matrix elements connecting the ground state with the excited states of each isomer. Our calculations were tested rigorously for convergence with respect to the basis set, as well as with respect to the size of the active orbital space employed in the CI calculations. These calculations reveal that as far as electron-correlation effects are concerned, core excitations play an important role in determining the optimized ground state geometries of various clusters, thereby requiring all-electron correlated calculations. But, when it comes to low-lying optical excitations, only valence electron correlation effects play an important role, and excellent agreement with the experimental results is obtained within the frozen-core approximation. For the case of $\mathrm{Na}_{6}$, the largest cluster studied in this work, we also discuss the possibility of occurrence of plasmonic resonance in the optical absorption spectrum.
\end{abstract}

\section{Introduction}

Clusters of atoms and molecules are considered to be strong candidates for developing future nanotechnological devices ranging from electron-transport based devices such as switches and transistors, to opto-electronic devices such as light-emitting diodes.[1] However, regardless of the nature of the application, in order to harness full potential of these atomic assemblies, it is important to achieve a deep theoretical understanding their electronic structure. $[2,3,4,5]$ Clusters can be seen as an intermediate state of matter lying somewhere between the atomic state, and the bulk state. Therefore, they

Department of Physics, Indian Institute of Technology Bombay, Powai, Mumbai 400076, INDIA e-mails: eccpradip@gmail.com,deepakrai@phy.iitb.ac.in, shukla@phy.iitb.ac.in 
can provide us important insights into the evolution of the matter from the microscopic to the macroscopic length scales. Of particular interest are issues such as the nature of chemical bonding, low-lying excited states, and the geometrical structure of the clusters as their size grows. One would intuitively expect that clusters whose bulk states are insulating, or semiconducting, will exhibit localized electrons. Likewise, electrons in clusters of atoms whose bulk form is metallic, will be expected to be itinerant. Indeed, in cluster science, an important direction of research is to understand as to what are the signatures of metallicity in metal atom clusters. $[2,3,4,5,6]$ If the electrons in the metal atom clusters are indeed metallic, can one use simple jellium model or some variant thereof, to describe their physics?[7,4] It is well known that the nature of low-lying optical excitation in bulk metals is plasmonic, that is collective. But what happens when we consider a metal atom cluster: is the nature of low-lying excited states for such systems molecular or collective?[7,4,8]

Alkali atoms have a rather simple electronic structure, with a single valence electron outside the closed shell. In the bulk form, alkali metals are considered to be a protypical examples of free-electron gas formed by the one electron contributed by each atom. Therefore, alkali atom clusters, will be the simplest systems on which the issue of metallicity in finite sized systems can be probed. Perhaps because of this simplicity that a number of theoretical and experimental probes of alkali atom clusters have been performed over the years. Early description of the electronic structure of clusters was performed in terms of the free-electron gas based jellium model, perhaps due to its general nature, and inherent simplicity. $[9,10,11]$ Jellium model was also employed to describe the photoabsorption in neutral and charged sodium clusters.[12] First principles methodologies were also used several years back to compute the optimized geometries, binding energies, and ionization potentials of small alkali metal clusters.[13, $14,15,16,17,18,19,20,21]$ Rao and Jena used first principles Hatree-Fock (HF) and configuration-interaction (CI) methodology to study the electronic structure, geometry, and magnetic properties of lithium based homo-atomic and hetero-atomic clusters.[14, 18] Martins et al. performed calculations employing first principles density-functional theory (DFT) within the local-spin-density approximation (LSDA) to study the ground state geometries and electronic structure of small sodium clusters.[15] First principles multi-reference configuration interaction (MRCI) approach was employed by Koutecký and coworkers to study the low-lying excited states and photoresponse of a number of lithium and sodium clusters.[16,17,19,22,20,13] Röthlisberger and Andreoni[23] used a first-principles molecular dynamics based approach to compute the structure and electronic properties of sodium clusters ranging from $\mathrm{Na}_{2}$ to $\mathrm{Na}_{20}$. Employing an $a b$ initio DFT based all electron approach, Blundell et al.[24] reported calculations of static polarizabilities of several Na clusters. Balbás performed time-dependent density functional theory (TDDFT) based calculations of the photoabsorption spectra of relatively large sodium clusters $\mathrm{Na}_{20}$ and $\mathrm{Na}_{40}$. [25] First principles approaches based upon HF, DFT, and second-order Møller-Plesset perturbation theory (MP2) were employed by Solov'yov et al.[26] to determine the geometry and other ground state properties of a number of sodium clusters. DFT based calculations on the electronic structure and geometry of the ground state of several neutral and cationic clusters of sodium were performed by Kümmel and et al.[27] An ab initio time-dependent local-density approximation (TDLDA) based methodology was adopted by Pacheco and Martins[28] to study the photoresponse of lithium and sodium clusters. First principles TDLDA was also employed by Moseler et al.[29] to compute the optical absorption spectra of cationic clusters of sodium. Gutiérrez et al. employed a first principles DFT based 
approach to compute the transport properties of small sodium clusters. Rubio and coworkers computed the optical absorption spectra of small sodium and silicon clusters using TDDFT based approaches.[30,31]TDDFT was also employed by Joswig et al.[32] to study the photoabsorption spectra of a large number of sodium clusters. Wang et al.[33] employed a TDDFT based approach to study plasmonic excitation in planar clusters of sodium. Recently, Pal et al. $[34,35]$ employed a first principles linear-response theory based Bethe-Salpeter equation approach to compute the optical absorption spectra of a number of closed-shell neutral and cationic sodium clusters.

Experimental measurements of photoabsorption processes in alkali metal clusters has been performed for a long time. Early experiments of photoabsorption in sodium and potassium dimers were performed by Fredrickson and Watson.[36] For the sodium trimer, optical absorption measurements have been reported by Herrmann et al.,[37] Wang et al.,[38] and Broyer et al.[39] As far as the spectroscopy of $\mathrm{Na}_{4}$ cluster is concerned, Wang et al. have again performed the measurements.[38,8] In a separate paper, the same group also reported the photoabsorption measurements in $\mathrm{Na}_{4}, \mathrm{Na}_{5}$, and $\mathrm{Na}_{7}$ clusters.[40] Schmidt and Haberland[41] measured the photoabsorption crosssections of sodium mono-cationic clusters ranging from $\mathrm{Na}_{3}{ }^{+}$to $\mathrm{Na}^{+}{ }_{64}$. Several measurements of photoabsorption cross-sections and static polarizabilities of sodium clusters have also been reported by de Heer and coworkers over the years. [42, 43, 44, 45, $6]$

As reviewed above, most of the calculations of the electronic structure and related properties of alkali metal clusters have been performed either using the jellium model, or, more recently, using the DFT based methodologies. Except for the early works of Rao and Jena,[14,18] and those of Koutecký and coworkers, [16, 17, 19,22,20,13,21] we are not aware of studies of the low-lying excited states and optical properties of alkali metal clusters which employ wave function based methodologies such as the CI approach, or the coupled-cluster (CC) method. While DFT based methodologies are certainly computationally very efficient because they can, in principle, include electroncorrelation effects at the mean-field level in the Kohn-Sham equations. However, the exact form of the exchange-correlation functional, which is supposed to incorporate these effects, is unknown. Therefore, the choice of the exchange-correlation functional $(\mathrm{XCF})$ is a matter of speculation, and as a result a huge array of such functionals are available. It is a well-known fact that results of calculations depend critically on the choice of the functional, and sometimes, one has to use different functionals to describe different properties of the same system. Some functionals provide a good description of the ground state geometry, while, others provide a better description of the cohesive energies. As far as the description of excited states of extended systems is concerned, at present the method of choice is TDDFT, in which a number of strides have been made recent times.[46] Nevertheless, TDDFT also suffers from the same problems as DFT, i.e., the results obtained are highly functional dependent. It also appears to be most effective in describing low-lying singly-excited states, while at higher energies, its accuracy appears to be questionable. For all these reasons we believe that it is very important to benchmark excited state results by performing accurate electroncorrelated first-principles calculations using the Born-Oppenheimer Hamiltonian, and wave-function-based methodologies. Because the Hamiltonian, unlike the functional is well-known, the only problem which wave-function-based methods experience are related to the truncation of the many-particle wave function. However, by systematically expanding the wave-function in terms of the many-particle basis, this problem can be curtailed. It is with this philosophy in mind that we have undertaken system- 
atic large-scale first-principles CI calculations of sodium clusters ranging from $\mathrm{Na}_{2}$ to $\mathrm{Na}_{6}$, to compute their linear photoabsorption spectra, and carefully compare our results to the spectroscopic data where available. In addition to the neutral clusters, calculations have also been performed for the closed-shell cationic clusters $\mathrm{Na}_{3}{ }^{+}$and $\mathrm{Na}_{5}{ }^{+}$. For several clusters, in addition to the global minima, we have also considered various possible low-lying geometries, with the intention of probing structure-property relationship which can serve as fingerprint for detection of cluster geometries using photo-absorption experiments. The computational methodology we have employed for the purpose is the frozen-core configuration interaction (CI) method at the full-CI or quadruple-CI level, which has been extensively used in our group in earlier studies of the optical properties of a number of conjugated polymers, $[47,48,49,50,51,52,53]$ and small boron and aluminum clusters. $[54,55,56]$ We also compare our results to the experimental ones where available, and find excellent agreement.

\section{Theoretical Background}

In this work we have adopted a first-principles wave function based methodology in which the molecular orbitals (MOs) are expanded as a linear combination of Cartesian Gaussian basis functions. Calculations are initiated by performing either the restricted Hartree-Fock (RHF), or the restricted open-shell Hartree-Fock (ROHF) calculations depending upon whether the cluster under consideration is electronically closed shell, or open-shell type, so as to obtain the MO basis set. Subsequently, correlated calculations are performed using the configuration-interaction (CI) methodology, and the approach is employed at various approximation levels such as singles-CI (SCI), full-CI (FCI), or quadruple-CI (QCI). For performing the CI calculations, we employed a configuration state function (CSF) basis, in which the CSFs were eigenfunctions of both the total spin $\left(S^{2}\right)$, as well as the $z$-component of the spin $\left(S_{z}\right)$. Furthermore, wherever possible, point group symmetries at the level of $D_{2 h}$ and its subgroups were also used to reduce the computational effort. We have employed this wave-function-based first principles approach in several of our recent works on clusters, $[55,57,56]$ and for performing these calculations we have used the MELD program package.[58]

As far as geometries of various isomers are concerned, in some cases they were obtained manually, by performing electron-correlated calculations for different geometries, while in other cases, automated geometry optimization at various levels of theory, as implemented in the Psi4 computer program,[59] was employed.[60,61] For a few isomers, we used the geometries reported by other authors, in previous papers.

Once the ground state geometry of an isomer is decided, ground and excited state calculations are performed on it using the CI methodology described in our previous works. $[47,48,49,50,51,54,52,55,56,53]$ The level of the CI approach adopted in the calculations is based upon the size of the isomer, as also the number of active electrons and orbitals. For the smaller isomers frozen-core FCI method was used, while for the larger ones, the frozen-core QCI approach was employed. For the purpose of benchmarking the frozen-core approximation, we employed the SCI method both at the all-electron, as well as frozen-core levels. Using the many-body wave functions obtained from the CI calculations, optical absorption spectrum was computed in the electric-dipole approximation, assuming a Lorentzian line shape. 


\section{Calculations and Results}

In this section, first we shall discuss the convergence of our calculations with respect to various approximations. Thereafter, the results of our calculations for various clusters will be presented with comprehensive discussion.

\subsection{Convergence of Calculations}

Here, we carefully examine the convergence of the calculated absorption spectra with respect to the size and quality of the basis set, along with various truncation schemes in the CI calculations.

\subsubsection{Choice of the basis set}

Because finite basis set is employed in electronic structure calculations, it is but natural that the results will depend upon the quality and the size of the basis set employed. Therefore, before comparing our results with the experiments, we want to explore the dependence of photo-absorption spectra on the basis set. It is obvious from the metallic nature of bulk sodium that its $3 \mathrm{~s}$ valence electron exhibits delocalized behavior, and, therefore, the basis set used for calculations should have diffuse Gaussian basis functions. We calculated the absorption spectra of $\mathrm{Na}_{2}$ employing basis sets $6-311++\mathrm{G}(3 \mathrm{df}, 3 \mathrm{pd}), 6-311++\mathrm{G}(2 \mathrm{~d}, 2 \mathrm{p})$, and also correlation-consistent basis sets aug-cc-pcVDZ and cc-pVTZ-DK, all of which have been taken from the EMSL Gaussian Basis Set Library. [60,61] Upon examining the calculated spectra presented in Fig. 1, the following trends emerge: the spectra computed by augmented basis sets 6 $311++\mathrm{G}(3 \mathrm{df}, 3 \mathrm{pd}), 6-311++\mathrm{G}(2 \mathrm{~d}, 2 \mathrm{p})$ and aug-cc-pcVDZ are in very good agreement with each other in the energy range examined, while the one computed by the set cc-pVTZ-DK is a bit blue shifted as far as higher energy peaks are concerned. Considering good agreement among three basis sets, we choose to perform calculations for all the isomers (unless, indicated otherwise) of sodium clusters using the basis set $6-311++\mathrm{G}(3 \mathrm{df}, 3 \mathrm{pd})$.

\subsubsection{Orbital Truncation Schemes}

It is a well-known fact that the computational effort in a CI calculation performed using $N$ MOs scales as $\approx N^{6}$, which can become quite unfavorable for large values of $N$. For first principles calculations utilizing large basis sets, generally $N$ tends to be large, and, therefore, it is very important to reduce the number of MOs used in the CI calculations. The number of occupied MOs is reduced by employing the so-called "frozen-core approximation" discussed earlier, while the number of unoccupied (virtual) states are truncated by ignoring high-energy MOs lying far from the Fermi level.

The influence of freezing the core orbitals on the optical absorption spectrum of $\mathrm{Na}_{2}$ cluster is displayed in Fig. 2, while for $\mathrm{Na}_{3}$ the results are presented in Fig. 3. It is obvious from the figures that it virtually makes no difference to the computed spectra when the core orbitals are frozen. The effect of removing the high-energy virtual orbitals on the absorption spectrum of clusters $\mathrm{Na}_{2}$ and $\mathrm{Na}_{4}$ are examined in Figs. 4 and 5, respectively. From these figures it is obvious that if the virtual orbitals above the energy of 1 Hartree are removed from the list of active orbitals, the absorption 


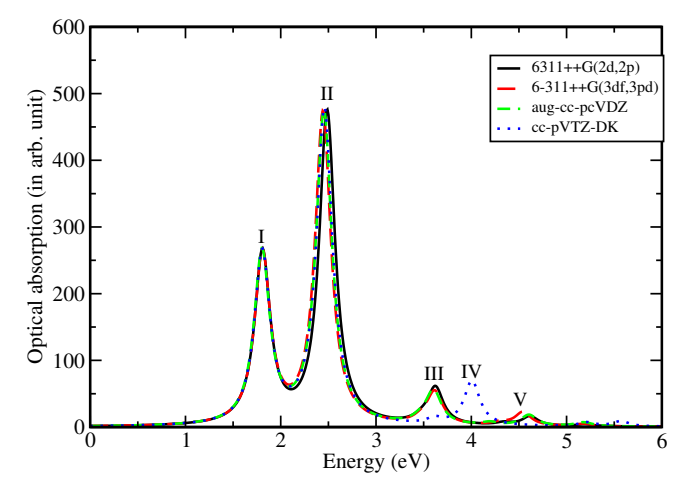

Fig. 1: Optical absorption in $\mathrm{Na}_{2}$ calculated using various Gaussian contracted basis sets.

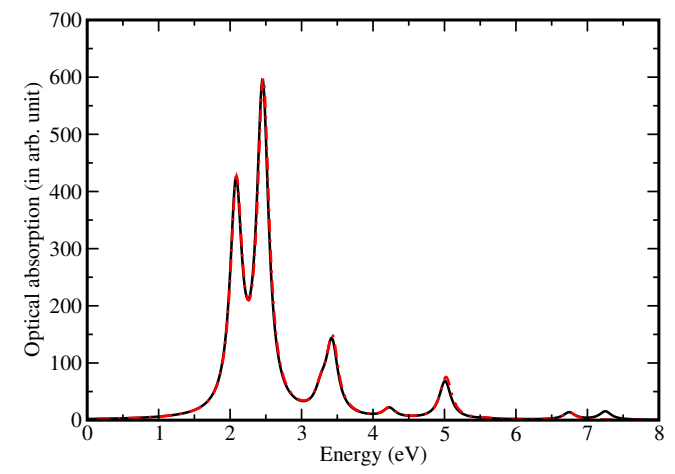

Fig. 2: (Color online) Comparison of the SCI level optical absorption spectrum of $\mathrm{Na}_{2}$ computed at the all-electron level ( in black), with that obtained using the frozen-core approximation (in red). Hardly any difference is visible between the two spectra.

spectrum stays unchanged. This is understandable on physical grounds because we are interested in computed absorption spectrum in the energy range much lower than 1 Hartree. Therefore, in rest of the calculations, all the core orbitals were frozen, and virtual orbitals with energies larger than this cutoff were not considered.

\subsubsection{Size of the CI expansion}

In CI calculations, greater numerical accuracy demands the inclusion of a rather large number of configurations which can make the calculations numerically intractable. 


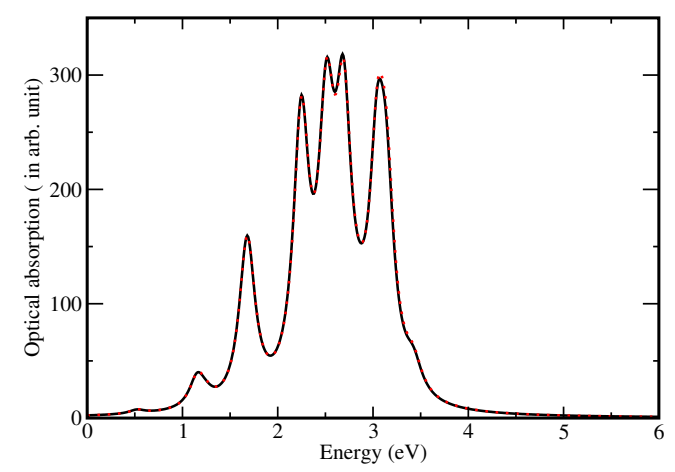

Fig. 3: (Color online) Same comparison as shown in Fig. 2, but for the case of $\mathrm{Na}_{3}$.

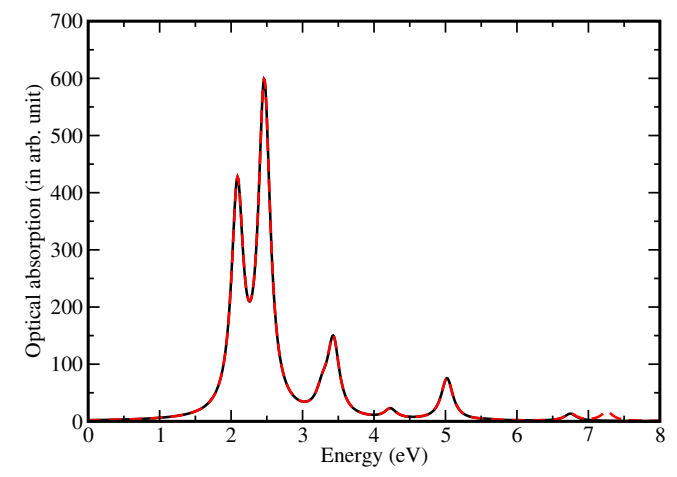

Fig. 4: (Color online) A comparison of all-electron SCI optical absorption spectrum of $\mathrm{Na}_{2}$ (in black), with that obtained by removing the virtual orbitals with energies larger than 1.0 Hartree (in red), from the list of active orbitals. The differences between two sets of calculations are insignificant.

However, when it comes to the computation of optical properties, our focus is on calculating accurate energy differences between the ground state, and the excited states, rather than their absolute energies, which is possible even with moderately large CI expansions. In Table 1 we present the average total number of configurations $\left(\mathrm{N}_{\text {total }}\right)$ for different isomers, with the average computed across different irreducible representations in these symmetry adapted calculations. The fact that these calculations employ more than three million configurations for the largest cluster considered ( $\left.\mathrm{Na}_{6}\right)$, makes us confident that our results are fairly accurate. 


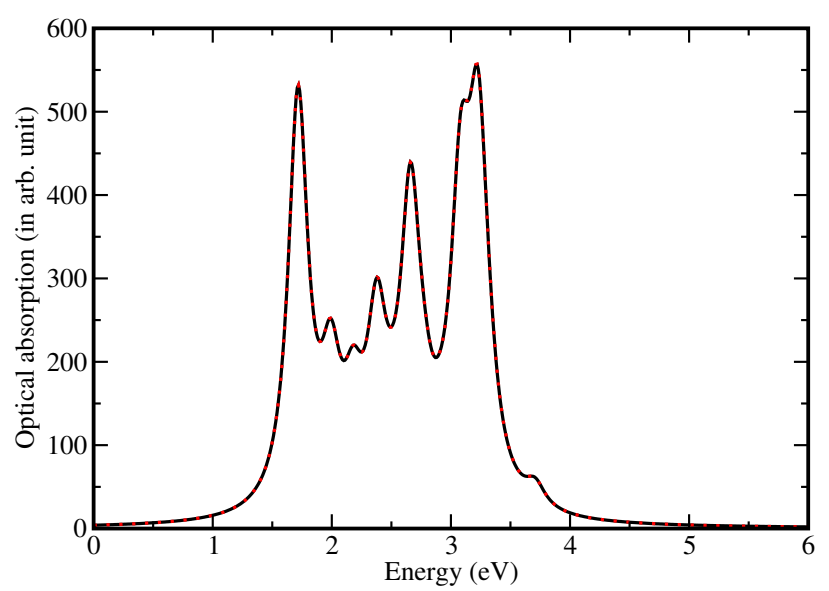

Fig. 5: (Color online) Same comparison as shown in Fig. 4, but for the case of $\mathrm{Na}_{4}$.

Before we discuss the absorption spectrum for each isomer, we present the ground state energies along with the relative energies of each isomer in Table 2, from which it can be easily deduced as to which isomer of a given cluster corresponds to the minimum energy ground state geometry. Furthermore, symmetry of the ground state wave function of each isomer is also listed in the same table.

\subsection{Calculated Absorption Spectra of Various Clusters}

We have performed calculations on various low-lying isomers of neutral sodium clusters $\mathrm{Na}_{n}$ with $n=2-6$, as well as on closed-shell cation clusters $\mathrm{Na}_{3}^{+}$and $\mathrm{Na}_{5}^{+}$. Optimized geometries of each of those isomers are depicted in Fig. 6.

Next we present and discuss the results of our calculations for different isomers.

3.2.1 $\mathrm{Na}_{2}$

Geometry optimization of sodium dimer was performed at the $\operatorname{CCSD}(\mathrm{T})$ level, employing Gaussian basis set 6-311++G(3df,3pd), taken from EMSL basis set library,[60, 61] and the equilibrium bond length was obtained at $3.07 \AA$ for the singlet ground state of symmetry ${ }^{1} \Sigma_{g}$ (cf. Fig. 6(a)). Pal et al. reported an equilibrium bond length of $3.30 \AA,[34]$ obtained at the HF level, which is about $0.23 \AA$ longer than our value. Based upon ab initio MP2 calculations Solov'yov et al. reported the bond length to be $3.153 \AA,[26]$ while Ozaki and Kino,[62] based upon first principles DFT calculations employing numerical orbitals obtained the value $3.175 \AA$. Martins et al.[15], reported somewhat shorter bond length of $2.91 \AA$. We note that our optimized bond length for 
Table 1: Average number of total configurations $\left(\mathrm{N}_{\text {total }}\right)$ involved in frozen-core CI calculations of optical absorption spectra of various isomers of sodium clusters.

\begin{tabular}{|c|c|c|c|}
\hline Cluster & Isomer & CI method & $\mathrm{N}_{\text {total }}$ \\
\hline \hline $\mathrm{Na}_{2}$ & Linear $\left(D_{\infty h}\right)$ & FCI & 7056 \\
\hline & & & \\
\hline $\mathrm{Na}_{3}{ }^{+}$ & Triangular $\left(D_{3 h}\right)$ & FCI & 992250 \\
\hline & & & \\
\hline $\mathrm{Na}_{3}$ & Triangular $\left(C_{2 v}\right)$ & FCI & 992250 \\
\hline & Linear $\left(D_{\infty h}\right)$ & FCI & 992250 \\
\hline & Equilateral $\left(D_{3 h}\right)$ & FCI & 992250 \\
\hline & & & \\
\hline $\mathrm{Na}_{4}$ & Rhombus $\left(D_{2 h}\right)$ & FCI & 725903 \\
\hline & Square $\left(D_{4 h}\right)$ & FCI & 1153510 \\
\hline & & & \\
\hline $\mathrm{Na}_{5}{ }^{+}$ & $D_{2 d}$ & FCI & 406456 \\
\hline & $D_{2 h}$ & FCI & 762300 \\
\hline & $D_{3 h}$ & FCI & 442176 \\
\hline & $C_{2 v}$ & FCI & 192660 \\
\hline $\mathrm{Na}_{5}$ & Planar $\left(C_{2 v}\right)$ & QCI & 3349740 \\
\hline & Bipyramid $\left(C_{2 v}\right)$ & QCI & 1463592 \\
\hline & & & \\
\hline $\mathrm{Na}_{6}$ & Planar $\left(D_{3 h}\right)$ & QCI & 3463230 \\
\hline & $C_{5 v}$ & QCI & 3463230 \\
\hline & $D_{4 h}$ & QCI & 3162995 \\
\hline
\end{tabular}

the sodium dimer is closer to values reported by Solov'yov et al.,[26] and Ozaki and Kino,[62] than that of Pal et al. [34]

Table 3: Comparison of location of calculated peaks in the photoabsorption spectrum of $\mathrm{Na}_{2}$, with the work of other authors, and experiments. Our calculations were performed using the frozen core FCI approach.

\begin{tabular}{|cccccccc|}
\hline \multirow{2}{*}{ Work } & \multirow{2}{*}{ Method } & \multirow{2}{*}{ Bond Length $(\AA)$} & \multicolumn{5}{c|}{ Peak Energies (Symmetry) $(\mathrm{eV})$} \\
\cline { 3 - 7 } & & & $\mathrm{I}\left({ }^{1} \Sigma_{u}\right)$ & $\mathrm{II}\left({ }^{1} \Pi_{u}\right)$ & $\mathrm{III}\left({ }^{1} \Pi_{u}\right)$ & $\mathrm{IV}\left({ }^{1} \Pi_{u}\right)$ & $\mathrm{V}\left({ }^{1} \Pi_{u}\right)$ \\
\hline This work & FCI & 3.07 & 1.87 & 2.48 & 3.66 & 4.62 & 5.46 \\
Theory (Ref.[34]) & BSE & 3.30 & 1.97 & 2.56 & 3.75 & & \\
Exp.(Ref.[36] $)$ & - & - & 1.86 & 2.52 & 3.75 & & \\
\hline
\end{tabular}

Using this bond length $(3.07 \AA)$, we computed the optical absorption spectrum of sodium dimer employing the FCI approach within the frozen-core approximation, which is presented in Fig. 7. The general qualitative features of the calculated spectrum are: (a) in the given energy range, five peaks are observed, with most of the oscillator strength contained in the first two peaks, (b) the first peak corresponds to a state of ${ }^{1} \Sigma_{u}$ symmetry, and is reached by a photon polarized along the molecular direction (longitudinal polarization), while the remaining peaks correspond to ${ }^{1} \Pi_{u}$ states, accessible by photons polarized perpendicular to the molecular direction (transverse polarization). Next we discuss the quantitative aspects of the spectrum.

The experimental absorption spectrum of $\mathrm{Na}_{2}$ measured by Fredrickson and Watson,[36] and reproduced in the paper of Pal et al.,[34] exhibits three peaks, whose intensity pro- 
Table 2: Ground state (GS) energies (in Hartree) and relative energies (in eV) corresponding to the geometries of various isomers depicted in Fig. 6, and the symmetry of their wave function. For all the cases except $\mathrm{Na}_{6}$ isomers, geometries were optimized using an all-electron CCSD(T) approach, as implemented in the Psi4 program,[59] employing the cc-PVTZ basis set.[61] For Na6 isomers, CCSD method[59] was utilized for geometry optimization, coupled with the cc-pvdz basis set.[61] In a few cases, previously published geometries, cited in the text, were used.

\begin{tabular}{|c|c|c|c|c|}
\hline Cluster & Isomer & Symmetry & GS energy & Relative energy \\
\hline & & & $(\mathrm{Ha})$ & $(\mathrm{eV})$ \\
\hline $\mathrm{Na}_{2}$ & Linear $\left(D_{\infty h}\right)$ & ${ }^{1} \Sigma_{g}$ & -323.7694 & 0.0000 \\
\hline $\mathrm{Na}_{3}{ }^{+}$ & Triangular $\left(D_{3 h}\right)$ & ${ }^{1} A_{1}^{\prime}$ & -485.5130 & 0.0000 \\
\hline \multirow[t]{3}{*}{$\mathrm{Na}_{3}$} & Triangular $\left(C_{2 v}\right)$ & ${ }^{2} B_{2}$ & -485.6519 & 0.0000 \\
\hline & Equilateral $\left(D_{3 h}\right)$ & ${ }^{2} A_{1}^{\prime}$ & -485.6490 & 0.07891 \\
\hline & Linear $\left(D_{\infty h}\right)$ & ${ }^{2} \Sigma_{u}$ & -485.6474 & 0.1225 \\
\hline \multirow[t]{2}{*}{$\mathrm{Na}_{4}$} & Rhombus $\left(D_{2 h}\right)$ & ${ }^{1} A_{g}$ & -647.5553 & 0.0000 \\
\hline & Square $\left(D_{4 h}\right)$ & ${ }^{1} A_{1 g}$ & -647.5360 & 0.5252 \\
\hline \multirow{4}{*}{$\mathrm{Na}_{5}{ }^{+}$} & $D_{2 d}$ & ${ }^{1} A_{1}$ & -809.3119 & 0.0000 \\
\hline & $D_{2 h}$ & ${ }^{1} A_{g}$ & -809.3117 & 0.0054 \\
\hline & $D_{3 h}$ & ${ }^{1} A_{1}^{\prime}$ & -809.3071 & 0.1306 \\
\hline & $C_{2 v}$ & ${ }^{1} A_{1}$ & -809.2882 & 0.6449 \\
\hline \multirow[t]{2}{*}{$\mathrm{Na}_{5}$} & Planar $\left(C_{2 v}\right)$ & ${ }^{2} A_{1}$ & -809.4505 & 0.0000 \\
\hline & Bipyramid $\left(C_{2 v}\right)$ & ${ }^{2} B_{1}$ & -809.4463 & 0.1143 \\
\hline \multirow[t]{3}{*}{$\mathrm{Na}_{6}$} & $C_{5 v}$ & ${ }^{1} A_{1}$ & -971.2509 & 0.0000 \\
\hline & Planar $\left(D_{3 h}\right)$ & ${ }^{1} A_{1}^{\prime}$ & -971.2476 & 0.0898 \\
\hline & $D_{4 h}$ & ${ }^{1} A_{1 g}$ & -971.2441 & 0.1850 \\
\hline
\end{tabular}

file is in excellent agreement with the first three peaks of our calculated spectrum ( $c f$. Fig. 7).The first absorption peak in our calculated spectrum is located $1.87 \mathrm{eV}$, which is in excellent agreement with the experimental value of $1.86 \mathrm{eV},[36]$ at which the absorption begins. Pal et al.[34] computed this peak to be located at $1.97 \mathrm{eV}$, which somewhat overestimates the experimental value. Wave functions and other information is presented in the Table 1 of Supporting Information, the many-particle wave function of the excited state corresponding to this peak is dominated by $|H \rightarrow L\rangle$ excitation, with $|H \rightarrow L+3\rangle$ excitation also making a significant contribution. The strongest peak in our calculated spectrum is the second one, located at $2.48 \mathrm{eV}$, which is also in excellent agreement with experimental value $2.52 \mathrm{eV}$ reported by Fredrickson and Watson.[36] Pal et al.[34] computed the location of this peak to be a bit higher at $2.56 \mathrm{eV}$. Wave function corresponding to this state exhibits strong configuration mixing with singly-excited configurations $\left|H \rightarrow(L+2)_{1}\right\rangle$ and $\left|H \rightarrow(L+2)_{2}\right\rangle$ (orbital $L+2$ belongs to $\pi_{u}$ irrep, and is doubly degenerate) making significant contributions. The third peak in the experimental absorption spectrum is much less intense as compared to the other peaks, and is located at $3.75 \mathrm{eV},[36,34]$ which is in good agreement with our calculated location of peak III at $3.66 \mathrm{eV}$. The excited state corresponding to this peak obtains its main contribution from the single excitation $|H \rightarrow L+2\rangle,|H \rightarrow L+9\rangle$, and 
$|H \rightarrow L+16\rangle$. Our calculations predict the existence of a couple of more faint peaks located at $4.62 \mathrm{eV}$, and 5.46, which have not been reported either in the experiment,[36] or in earlier theoretical works. The wave function of the corresponding excited states are a strong mixture of higher-energy single excitation, and a few doubly excited configurations. It will be interesting to see if future experiments on sodium dimer are able to detect these higher energy peaks, predicted in this work.

\subsection{2 $\mathrm{Na}_{3}{ }^{+}$}

Next, we discuss the optical absorption spectrum of cation $\mathrm{Na}_{3}^{+}$, which, under the frozen-core approximation, has only two active electrons similar to the case of sodium dimer. The geometry of the system is well known to be an equilateral triangle with point group $D_{3 h}$, so that the electronic ground state corresponds to the symmetry ${ }^{1} A_{1}^{\prime}$, while optically active excited states belong to ${ }^{1} E^{\prime}$ symmetry, achievable by photons polarized in the plane of the molecule, and ${ }^{1} A_{2}^{\prime \prime}$ achievable through photons polarized perpendicular to the plane of the triangle. When we optimized the geometry of this cluster by using the $6-311++\mathrm{G}(3 \mathrm{df}, 3 \mathrm{pd})$ contracted Gaussian basis set at the full CI level, assuming frozen cores, the optimized bond lengths of the triangle were obtained to be $3.58 \AA$, which is smaller by $0.06 \AA$ as compared to the bond length obtained by Pal et al.[34] Using that bond length, in our calculated spectrum, we obtain two dominant peaks at $2.52 \mathrm{eV}\left({ }^{1} E^{\prime}\right)$, and $3.15 \mathrm{eV}\left({ }^{1} A_{2}^{\prime \prime}\right)$, which are in reasonable agreement with the experimentally reported peaks by Schmidt et al.[41] at $2.62 \mathrm{eV}$, and $3.33 \mathrm{eV}$, respectively. However, Pal et al.,[34] using their BSE based approach, report a much better agreement with the experiments with the computed locations of the peaks at 2.64, and $3.36 \mathrm{eV}$, respectively. But, previous theoretical work of Bonačić-Kouteckỳ et al.,[21] reports much smaller optimized bond length $3.393 \AA$ for the ground state, therefore, we decided to probe the influence of geometry on optical absorption. We performed geometry optimization of the system by going beyond the frozen-core approximation, employing the CCSD(T) approach as implemented in the Psi4 code.[59] Furthermore, we also probed the influence of the basis set on the photoabsorption spectrum of this system, and the results of this investigation are summarized in Table 4 . Our best results for the absorption spectrum correspond to peak locations $2.62 \mathrm{eV}$ and $3.25 \mathrm{eV}$, and were obtained from frozen-core FCI calculations performed using $6-311++\mathrm{G}(3 \mathrm{df}, 3 \mathrm{pd})$ basis set, and an optimized bond length of $3.394 \AA$ obtained from $\operatorname{CCSD}(\mathrm{T})$ based optimization. We note that: (a) our optimized bond length is significantly shorter as compared to the value obtained by Pal et al.,[34] but is in excellent agreement with the value obtained by Bonačić-Kouteckỳ et al.,[21] and (b) our calculated location of the first peak is in perfect agreement with the experiments, while the second one is underestimated by $0.08 \mathrm{eV}$.

As far as the single-particle energy levels of the system are concerned, the HOMO of the cluster, denoted as $H$, is non-degenerate, while the LUMO levels, denoted $L_{1}$ and $L_{2}$, exhibit a two-fold degeneracy. Wave functions and other information is presented in the Table 2 of Supporting Information, many-particle wave functions of the states giving rise to the peaks observed in the photoabsorption spectrum are presented. From there it is obvious that the first peak, which corresponds to a doubly degenerate state, has strong contributions from singly excited configurations $\left|H \rightarrow L_{1}\right\rangle$ and $\left|H \rightarrow L_{2}\right\rangle$, while the wave function of the second one is dominated by $|H \rightarrow L+1\rangle$ single excitation. As compared to earlier studies, in our work, we observe two high-energy peaks (lableld III and IV in Fig. 8) near $5.5 \mathrm{eV}$. Peak III located at $5.45 \mathrm{eV}$ belongs to an excited state 
of ${ }^{1} E^{\prime}$ symmetry, while peak IV located at 5.59 is due to a state of symmetry ${ }^{1} A_{2}^{\prime \prime}$. The many-particle wave functions of these states are dominated by single exacitations from the HOMO to higher unoccupied orbitals ( $c f$. Table 2 of Supporting Information). These higher energy peaks predicted by our calculations can be explored in future experiments performed on this cluster.

Table 4: Comparison of location of peaks in the photoabsorption spectrum of $\mathrm{Na}_{3}^{+}$calculated using various basis sets and optimized geometries, with the theoretical works of other authors, and also with experimental results. All our calculations were performed using the frozen core FCI approach.

\begin{tabular}{|ccccc|}
\hline \multirow{2}{*}{ Work } & \multirow{2}{*}{ Method } & \multirow{2}{*}{ Bond Length $(\AA)$} & \multicolumn{2}{c|}{ Peak Energies (Symmetry) (eV) } \\
\cline { 4 - 5 } & & & $\mathrm{I}\left({ }^{\top} E^{\prime}\right)$ & $\mathrm{II}\left({ }^{\perp} A_{2}^{\prime \prime}\right)$ \\
\hline This work $^{a}$ & FCI & 3.580 & 2.52 & 3.15 \\
This work $^{b}$ & FCI & 3.435 & 2.60 & 3.23 \\
This work $^{c}$ & FCI & 3.394 & 2.60 & 3.23 \\
This work $^{d}$ & FCI & 3.394 & 2.62 & 3.25 \\
Theory (Ref.[21]) $_{\text {Theory (Ref.[34]) }}$ & FCI & 3.393 & 2.65 & 3.37 \\
Exp.(Ref.[41]) & BSE & 3.640 & 2.64 & 3.36 \\
& - & - & 2.62 & 3.33 \\
\hline
\end{tabular}

${ }^{a}$ Geometry optimization performed using the frozen core FCI technique, and 6$311++\mathrm{G}(3 \mathrm{df}, 3 \mathrm{pd})$ basis set for all calculations

${ }^{b}$ Geometry optimization performed using the $\operatorname{CCSD}(\mathrm{T})$ method using the $6-311++\mathrm{G}(3 \mathrm{df}, 3 \mathrm{pd})$ basis set employed for all calculations

${ }^{c}$ Geometry optimization performed using the $\operatorname{CCSD}(\mathrm{T})$ method with CC-VTZ basis set employed for all calculations

${ }^{d}$ Geometry optimization performed using the CCSD(T) method with CC-VTZ basis set, and excited state calculations performed using $6-311++\mathrm{G}(3 \mathrm{df}, 3 \mathrm{pd})$ basis set.

\section{$3.2 .3 \mathrm{Na}_{3}$}

Next, we discuss optical absorption spectrum of $\mathrm{Na}_{3}$, which is an open-shell system, with three active electrons, under the frozen-core approximation. The cluster possesses a symmetry broken ground state of an isosceles triangle with point group $C_{2 v}$, as against an equilateral triangular geometry of $\mathrm{Na}_{3}^{+}$cluster. In our calculations, the electronic ground state of the system was obtained to be ${ }^{2} B_{2}$ in agreement with previous theoretical results.[17] As per dipole selection rules of $C_{2 v}$ point group, with the ${ }^{2} B_{2}$ symmetry of the ground state, linear optical absorption can take place to the excited states of symmetries ${ }^{2} A_{1}$ (polarized perpendicular to the plane), ${ }^{2} A_{2}$ (inplane, short-axis, polarized), and ${ }^{2} B_{2}$ (in-plane, long-axis polarized). As far as bond lengths of the cluster are concerned, several values differing significantly from each other have been reported in the literature. For example, Martins et al.,[15] based upon first-principles molecular dynamics approach, obtained optimized bond lengths of 3.016 $\AA$ (equal arms) and $3.757 \AA$ (base), while Calaminici et al.[63] using first principles DFT reported lengths $3.170 \AA$ and $4.226 \AA$ for the same bonds. Bonačić-Kouteckỳ et $a l .,[17,64]$ using first-principles Hartree-Fock approach, reported two different sets of bond lengths in two papers: (a) $3.42 \AA$ and $4.79 \AA$,[17] and (b) $3.372 \AA$ and 4.509 
A.[64] Therefore, we performed our own geometry optimization for this cluster employing CCSD method with 6-311++G(3df,3pd) basis set, and obtained values $3.249 \AA$ and $4.285 \AA$, which are in reasonably good agreement with those reported by Calaminici et al.[63] In order to understand the influence of geometry on the photoabsorption spectrum, we decided to compute it for several of these geometries, and the results of the calculations performed using frozen-core FCI method and $6-311++\mathrm{G}(3 \mathrm{df}, 3 \mathrm{pd})$ basis set, are presented in Table 5 . In the same table, we also present the location and symmetries of optical absorption peaks of $\mathrm{Na}_{3}$ measured by Wang et al.,[38] using molecular beam photodepletion approach, while our absorption spectrum computed using the geometry of Calaminici et al.[63] is presented in Fig. 9.

In the measured spectrum of $\mathrm{Na}_{3}$, Wang et al.[38] identified the symmetry of the ground state as the ${ }^{2} B_{2}$, along with seven peaks due to linear optical absorption from the ground state, located at $1.67 \mathrm{eV}\left({ }^{2} A_{1}\right), 1.84 \mathrm{eV}\left({ }^{2} A_{2}\right), 2.03 \mathrm{eV}\left({ }^{2} A_{1}\right), 2.22 \mathrm{eV}$ $\left({ }^{2} B_{2}\right), 2.40 \mathrm{eV}\left({ }^{2} B_{2}\right), 2.59 \mathrm{eV}\left({ }^{2} A_{2}\right)$, and $3.00 \mathrm{eV}\left({ }^{2} A_{2}\right)$, where, in the parenthesis the symmetry of the corresponding excited state is indicated. Upon comparing this with the results of our calculations presented in Table 5, we conclude: (a) peak locations predicted by our calculations are in very good agreement with the experimental values of Wang et al.[38], except for the peak of symmetry ${ }^{2} B_{2}$ located at $1.84 \mathrm{eV}$, which is found in none of our calculations, (b) our calculations, as well as those of Bonačić-Kouteckỳ et al.,[64] predict two additional weak peaks of symmetry ${ }^{2} A_{1}$ located near $0.5 \mathrm{eV}$ and $1.1 \mathrm{eV}$ (labeled I and II, respectively) which are not observed in the experiment, and (c) we obtain the best agreement with the experiments using the geometry reported by Calaminici et al.,[63] although results obtained using other geometries are also fairly close. As far as intensity profile is concerned, our best calculation predicts that the highest intensity peak (peak VII in Fig. 9) is located at $2.53 \mathrm{eV}$ and corresponds to a state of symmetry ${ }^{2} A_{2}$, which is in excellent agreement with the experimental results of Wang et al.[38] who also assign symmetry ${ }^{2} A_{2}$ to their most intense peak, located at $2.59 \mathrm{eV}$. Therefore, we conclude that our calculated optical absorption spectrum of isosceles triangle shaped $\mathrm{Na}_{3}$ cluster is in excellent agreement with the experimental results.[38] Wave functions and other information is presented in the Table 3 of Supporting Information, peak I derives its main contribution $H \rightarrow L$ single excitation, and all the peaks are dominated by states which are mainly composed of singly excited configurations.

Table 5: Comparison of location of peaks in the photoabsorption spectrum of $\mathrm{Na}_{3}$ isosceles triangular cluster, calculated using various basis sets and optimized geometries, with experimental results. All photoabsorption calculations were performed using the frozen core FCI approach.

\begin{tabular}{|c|c|c|c|c|c|c|c|c|c|c|c|}
\hline \multirow{2}{*}{ Work } & \multirow{2}{*}{ Method } & \multirow{2}{*}{ Bond Length $(\AA)$} & \multicolumn{9}{|c|}{ Peak Energies (Symmetry) (eV) } \\
\hline & & & $\mathrm{I}\left({ }^{2} A_{1}\right)$ & $\mathrm{II}\left({ }^{2} A_{1}\right)$ & $\mathrm{III}\left({ }^{2} A_{1}\right)$ & $\left({ }^{2} B_{2}\right)$ & $\operatorname{IV}\left({ }^{2} A_{1}\right)$ & $\mathrm{V}\left({ }^{2} B_{2}\right)$ & $\mathrm{VI}\left({ }^{2} B_{2}\right)$ & $\mathrm{VII}\left({ }^{2} A_{2}\right)$ & V] \\
\hline This work $^{a}$ & FCI & $3.170,4.226$ & 0.50 & 1.11 & 1.61 & & 1.99 & 2.20 & 2.35 & 2.53 & \\
\hline This work ${ }^{b}$ & FCI & $3.016,3.757$ & 0.33 & 1.18 & 1.57 & & 2.04 & 2.25 & & 2.55 & \\
\hline This work ${ }^{c}$ & FCI & $3.249,4.285$ & 0.48 & 1.09 & 1.58 & & 1.96 & 2.16 & 2.33 & 2.51 & \\
\hline This work $^{d}$ & FCI & $3.372,4.509$ & 00.51 & 1.05 & 1.56 & & 1.93 & 2.11 & 2.29 & 2.48 & \\
\hline Exp.(Ref.[38]) & - & - & & & 1.67 & 1.84 & 2.03 & 2.22 & 2.40 & 2.59 & \\
\hline
\end{tabular}

${ }^{a}$ Geometry taken from Ref.[63], and excited state calculations performed using 6$311++\mathrm{G}(3 \mathrm{df}, 3 \mathrm{pd})$ basis set. 
${ }^{b}$ Geometry taken from Ref.[15], and excited state calculations performed using 6 $311++\mathrm{G}(3 \mathrm{df}, 3 \mathrm{pd})$ basis set.

${ }^{c}$ Geometry optimization performed using the CCSD method with 6-311++G(3df,3pd) basis set, and excited state calculations performed using $6-311++\mathrm{G}(3 \mathrm{df}, 3 \mathrm{pd})$ basis set.

${ }^{d}$ Geometry taken from Ref.[13], and excited state calculations performed using 6$311++\mathrm{G}(3 \mathrm{df}, 3 \mathrm{pd})$ basis set.

We have also computed the optical absorption spectrum of sodium trimer, with equilateral triangular geometry. Geometry optimization was performed by CCSD(T) method using the $6-311++\mathrm{G}(3 \mathrm{df}, 3 \mathrm{pd})$ basis set, and the optimized bond length was obtained $3.38 \AA$. Photoabsorption spectrum computed using this optimized bond length, and the frozen-core FCI approach, is presented in Fig. 10, and exhibits six peaks located at $1.19 \mathrm{eV}, 2.07 \mathrm{eV}, 2.36 \mathrm{eV}, 2.96 \mathrm{eV}, 3.72 \mathrm{eV}$, and $3.96 \mathrm{eV}$. On comparing the photoabsorption spectra of isosceles and equilateral triangles presented in Figs. 9 and 10, we note significant qualitative and quantitative differences between them which should allow one to differentiate between the two geometries using absorption-based experiments. Wave functions and other information is presented in the Table 4 of Supporting Information, we find that the first three peaks in the absorption spectrum of the equilateral triangular cluster correspond to photons polarized in plane of the molecule, in complete contrast with the results for the isosceles triangular cluster ( $c f$. Table 3 of Supporting Information). As far as the wave functions of excited states contributing to the peaks are concerned ( $c f$. Table 4 of Supporting Information), first four peaks consist of states whose wave functions are dominated by single excitations, while wave functions of those contributing to last two peaks derive main contributions from double excitations.

Apart from triangular geometries, we have also performed the optical absorption calculations for linear $\mathrm{Na}_{3}$ for which the ground state has optimized bond length 3.41 $\AA$ with symmetry ${ }^{2} \Sigma_{u}$, of symmetry group $D_{\infty h}$. According to dipole selection rules of group $D_{\infty h}$, optical absorption will take place to excited states of symmetries ${ }^{2} \Sigma_{g}$, polarized along the length of the molecule, and doubly-degenerate ${ }^{2} \Pi_{g}$ states, polarized in a plane perpendicular to the molecular axis. Calculated photoabsorption spectrum shown in Fig. 11 exhibits three peaks located at $0.64 \mathrm{eV}\left({ }^{2} \Sigma_{g}\right), 1.34 \mathrm{eV}\left({ }^{2} \Sigma_{g}\right)$ and $2.43 \mathrm{eV}\left({ }^{2} \Pi_{g}\right)$, of which the first peak is a rather weak one. Wave functions and other information is presented in the Table 5 of Supporting Information, we note that for the first two peaks, the wave functions are dominated by singly excited configurations, while the third peak is doubly degenerate and wave functions are dominated by both singly and doubly excited configurations. Strong configuration mixing exhibited by wave functions of excited states contributing to peak III, points to the importance of electron-correlation effects in the description of higher energy states.

\section{$3.2 .4 \mathrm{Na}_{4}$}

Geometry optimization for this cluster was performed using 6-311++G(3df,3pd) contracted Gaussian basis set, using the $\operatorname{CCSD}(\mathrm{T})$ approach implemented in Psi4 computer program. [59] The resultant rhombus-shaped equilibrium geometry shown in Fig. 6(f) is almost identical to those reported by Solovyov et al.,[26] and Pal et al.[34] The ground state belongs to the ${ }^{1} A_{g}$ irreducible representation of the $D_{2 h}$ point group, so that the excited states visible in the linear optical absorption will correspond to the symmetries ${ }^{1} B_{1 u}$ (out of plane polarized), ${ }^{1} B_{2 u}$ (short-diagonal polarized) and ${ }^{1} B_{3 u}$ (long-diagonal polarized). Optical absorption calculations were performed at the frozen-core FCI level 
using the same 6-311++G(3df,3pd) basis set, and the results are presented in Fig. 12 . Wang et al.. [38,8] measured the optical absorption spectrum of this cluster using the molecular beam photodepletion approach, and found eight absorption peaks located at: $1.63 \mathrm{eV}\left({ }^{1} B_{2 u}\right), 1.80 \mathrm{eV}\left({ }^{1} B_{3 u}\right), 1.98 \mathrm{eV}\left({ }^{1} B_{3 u}\right), 2.18 \mathrm{eV}\left({ }^{1} B_{1 u}\right), 2.51 \mathrm{eV}\left({ }^{1} B_{2 u}\right)$, 2.63-2.78 eV $\left({ }^{1} B_{1 u}\right), 2.85-3.15 \mathrm{eV}\left({ }^{1} B_{1 u},{ }^{1} B_{3 u}\right), 3.33 \mathrm{eV}\left({ }^{1} B_{2 u}\right)$, out of which the ones at $1.80 \mathrm{eV}$ and $2.51 \mathrm{eV}$ are the most intense. Our calculated spectrum (cf. Fig. 12) shows several peaks out of which the locations and the symmetries of the first eight are: $1.71 \mathrm{eV}\left({ }^{1} B_{3 u}\right), 2.09 \mathrm{eV}\left({ }^{1} B_{1 u}\right), 2.47 \mathrm{eV}\left({ }^{1} B_{2 u}\right), 2.69 \mathrm{eV}\left({ }^{1} B_{1 u}\right), 3.01 \mathrm{eV}\left({ }^{1} B_{1 u}\right)$, $3.22 / 3.28 \mathrm{eV}\left({ }^{1} B_{2 u} /{ }^{1} B_{1 u}\right), 3.90 \mathrm{eV}\left({ }^{1} B_{1 u}\right)$, and $4.01 \mathrm{eV}\left({ }^{1} B_{1 u}\right)$, out of which the peaks $1.71 \mathrm{eV}$ and $2.47 \mathrm{eV}$ are the most intense. Thus, on comparing our theoretical results with the experimental ones, $[38]$ we find that the location and symmetries of the two most intense peaks are reproduced excellently by our calculations. Furthermore, our weaker peaks located at $2.69 \mathrm{eV}, 3.01 \mathrm{eV}, 3.22 \mathrm{eV}$ compare very well with experimental peaks at $2.63-2.78 \mathrm{eV}, 2.85 \mathrm{eV}, 3.33 \mathrm{eV}$, both in locations and symmetries. On three weak peaks there is disagreement between the theory and the experiments: (a) the experimental absorption starts with a weak peak at $1.63 \mathrm{eV}\left({ }^{1} B_{2 u}\right)$ which is absent in our calculations, and (b) experimental spectrum observes couple of weak peaks corresponding to ${ }^{1} B_{3 u}$ symmetry states located at $1.98 \mathrm{eV}$ and $3.15 \mathrm{eV}$, which are absent in our spectrum. Thus, out of eight experimental peaks,[38] our theoretical calculations are able to resolve five peaks quite well, as far as their energetic location, point group symmetries, and intensities are concerned. Wave functions and other information is presented in the Table 6 of Supporting Information. From the table it is obvious that the wave functions of the excited states behind both the two intense peaks located at $1.71 \mathrm{eV}$ and $2.47 \mathrm{eV}$ are dominated by single excitation. The peak at $1.71 \mathrm{eV}$ derives main contributions from the excitation $|H \rightarrow L+2\rangle$ and $|H \rightarrow L+10\rangle$, while the one at $2.47 \mathrm{eV}$ is dominated by four configurations $|H \rightarrow L+6\rangle,|H \rightarrow L+18\rangle,|H \rightarrow L\rangle$, and $|H \rightarrow L+16\rangle$.

We also performed the optical absorption calculations for square-shaped $\mathrm{Na}_{4}$ cluster, employing the frozen-core FCI approach, and the results are presented in Fig. 13, along with the excited wave functions in Table 7 of Supporting Information. For these calculations, 6-311++G(3df,3pd) basis set was used, along with bond length $3.48 \AA$, which was obtained using all-electron $\operatorname{CCSD}(\mathrm{T})$ geometry optimization.[59] The ground state geometry corresponds to symmetry ${ }^{1} A_{1 g}$ of the $D_{4 h}$ point group, therefore, linear absorption, as per dipole selection rules, will be to excited states of symmetries ${ }^{1} E_{u}$ (in-plane polarized), and ${ }^{1} A_{2 u}$ (perpendicularly polarized). Wave functions and other information is presented in the Table 7 of Supporting Information, first four absorption peaks correspond to doubly degenerate ${ }^{1} E_{u}$ symmetry located at $1.16 \mathrm{eV}, 2.01$ $\mathrm{eV}, 2.17 \mathrm{eV}$, and $2.48 \mathrm{eV}$, while the fifth peak located at $2.75 \mathrm{eV}$ corresponds to an excited state of symmetry ${ }^{1} A_{2 u}$. This result is qualitatively distinct as compared to the case of rhombus ( $c f$. Fig. 12), for which, out of the first four absorption peaks, two were polarized perpendicular to the plane of the rhombus. Another interesting aspect of the first peak of square-shaped cluster is that the excited states giving rise to it derive significant contributions from double excitations ( $c f$. Table 7 of Supporting Information), while for the rhombus cluster, the wave function of the first optically active state is dominated by single excitations ( $c f$. Table 6 of Supporting Information). Furthermore, compared to the rhombus shaped cluster, optical absorption spectrum of the square-shaped cluster has relatively lesser number of features, and also different excitation energies for various peaks. Additionally, the first peak of the rhombus is the most intense one of its spectrum, while the first one of the square is the least intense 
one of the corresponding absorption spectrum Therefore, peak locations, along with the intensity profiles of the photoabsorption spectra of the two isomers can be used to distinguish them using optical spectroscopy.

\subsection{5 $\mathrm{Na}^{+}$}

For $\mathrm{Na}_{5}^{+}$, we have considered four different isomers with symmetries $D_{2 d}, D_{2 h}, D_{3 h}$, and $C_{2 v}$, as shown in Figs. $6(\mathrm{~h})-6(\mathrm{k})$, respectively. Various bond lengths corresponding to the $D_{2 d}, D_{2 h}$ and $D_{3 h}$ isomers indicated in those figures were obtained by performing geometry optimization using all-electron $\operatorname{CCSD}(\mathrm{T})$ approach implemented in the Psi4 program,[59] while for the $C_{2 v}$ isomer we used the geometry reported by $\mathrm{Pal}$ et at.[34]. We note that Pal et al. considered a $C_{4 v}$ isomer instead of $D_{2 h}$, however, our geometry optimization program led to the $D_{2 h}$ structure even when we initialized it with the $C_{4 v}$ geometry parameters of Pal et al. Although, our geometry optimization predicts the $D_{2 d}$ isomer to be the lowest energy structure, but the total CCSD(T) energies (cf. Table 2) of all the four isomers are very close to each other, with $D_{2 d}$ and $D_{2 h}$ energies being nearly degenerate, while energies of $D_{3 h}$ and $C_{2 v}$ isomers being just $0.13 \mathrm{eV}$, and $0.64 \mathrm{eV}$, respectively, higher than that of the $D_{2 d}$ isomer. Given the energetic proximities of these isomers, there is a high probability that in room temperature optical absorption experiments, absorption spectra of several of these isomers could be observed. Next, we discuss the calculated absorption spectra of each of these isomers.

In order to compute the optical absorption spectra, for all isomers, we used the $6-311++\mathrm{G}(3 \mathrm{df}, 3 \mathrm{pd})$ basis set coupled with the frozen-core FCI approach, and results of these calculations are presented in Fig. 14. Schmidt et al. $[41,65,66]$ performed experimental measurements of the optical absorption spectrum of this cluster, and found three absorption peaks located at: $2.2 \mathrm{eV}, 2.7 \mathrm{eV}$ and $3.3 \mathrm{eV}$. As far as the intensity pattern is concerned, the measured spectrum consists of three high intensity peaks, first one of which is the most intense, while the last one carries the least intensity of the three.[41] If we compare the intensity profiles of our theoretical spectra with the measured one, we find perfect agreement between computed spectra of isomers of $D_{2 d}$ and $D_{2 h}$ symmetries, and the experiment. In Table 6 we present the peak locations of the calculated spectra and the experimental one, and find that the excitation energies of three major peaks in the calculated spectrum of $D_{2 d}$ isomer are in excellent agreement with the experiments. We note that the first peak in the calculated spectrum of $D_{2 d}$ isomer located at $1.77 \mathrm{eV}$ is an extremely weak one, and has not been observed in the experiments. Furthermore, the peak locations of the first and the third peaks in the calculated spectrum of the $D_{2 h}$ symmetry isomer are also in very good experiment with the experiments, while that of the second peak is underestimated by about 0.19 $\mathrm{eV}$. Additionally, from Table 6 it is obvious that it is location of the second major peak which is sensitive to the geometry of the isomer, while locations of the first and third peaks for all the isomers are in reasonable agreement with the experiments. Because, for the $D_{2 d}$ isomer even the location of the second intense peak $(2.67 \mathrm{eV})$ is in very good agreement with the experimental value $(2.70 \mathrm{eV})$, we believe that the experimental result corresponds to the absorption spectrum of this isomer.

Detailed information about the excitation energies, symmetries, wave functions, polarizations, and oscillator strengths corresponding to various optical transitions of these isomers is presented in Tables 8-11 of the Supporting Information. We find that 
wave functions of most of the optically active excited states are dominated by singlyexcited configurations.

Bonačić-Kouteckỳ et al.,[21] and Pal et at.[34] also performed theoretical calculations of the optical absorption spectra of various isomers of $\mathrm{Na}_{5}^{+}$cluster, and in agreement with our work, they also obtained the lowest energy structure to be the isomer with $D_{2 d}$ symmetry. Various peak positions obtained by Pal et at.[34] are in very good quantitative agreement with our work. As far as CI method based calculations of Bonačić-Kouteckỳ et al.,[21] are concerned, we not only have good quantitative agreement on the peak locations, additionally, symmetries of the three intense peaks match perfectly with our work. Additionally, in their calculations,[21] the first peak is a faint one located below $2 \mathrm{eV}$, in agreement with our first weak peak of the $D_{2 d}$ isomer located at $1.77 \mathrm{eV}$.

Table 6: Comparison of location of peaks in the photoabsorption spectrum of $\mathrm{Na}_{5}{ }^{+}$ cluster for different isomers, calculated using $6-311++\mathrm{G}(3 \mathrm{df}, 3 \mathrm{pd})$ basis set and optimized geometries, with experimental results. All photoabsorption calculations were performed using the frozen core FCI approach.

\begin{tabular}{|c|c|c|c|c|c|c|c|}
\hline \multirow{2}{*}{ Work } & \multirow{2}{*}{ Method } & \multirow{2}{*}{ Isomers } & \multicolumn{5}{|c|}{ Peak Energies (Symmetry) $(\mathrm{eV})$} \\
\hline & & & $\mathrm{I}$ & II & III & IV & $\bar{V}$ \\
\hline This work & FCI & $D_{2 h}$ & $2.15\left({ }^{1} B_{2 u}\right)$ & $2.51\left({ }^{1} B_{3 u}\right)$ & $3.22\left({ }^{1} B_{1 u}\right)$ & $3.63\left({ }^{1} B_{3 u}\right)$ & \\
\hline This work & FCI & $C_{2 v}$ & $2.10\left({ }^{1} B_{2}\right)$ & $2.40\left({ }^{1} A_{1}\right)$ & $3.21\left({ }^{1} B_{1}\right)$ & & \\
\hline Exp.(Ref.[41,65,66]) & - & - & 2.20 & 2.70 & 3.30 & & \\
\hline
\end{tabular}

\subsection{6 $\mathrm{Na}_{5}$}

For $\mathrm{Na}_{5}$, which is an open-shell system, we have considered two isomers, both with $C_{2 v}$ symmetry: a planar structure shown in Fig. 6(l), and a trigonal bipyramidal structure shown in Fig. 6(m). We performed geometry optimization for the planar isomer, by employing all-electron CCSD approach implemented in the Psi4 code,[59] and the geometrical parameters obtained are indicated in Fig. 6(1). For the bipyramid structure we used the geometry reported in paper by Bonačić-Kouteckỳ et al.,[67] and we find the total energy of this structure to be only $0.11 \mathrm{eV}$ higher than that of the planar one $(c f$. Table 2). Our calculated optical absorption spectra for both the isomers is presented in Fig. 15, and it shows several absorption peaks ranging from $1.15 \mathrm{eV}$ to $2.76 \mathrm{eV}$, as summarized in Table 7. Out of these peaks, for the planar cluster, strong absorption peaks fall in the range 1.96-2.76 eV, while for the bipyramidal isomer, they lie in the range 2.02-2.44 eV. Experimental measurement of photoabsorption spectrum of $\mathrm{Na}_{5}$ cluster by Wang et al.,[40] reports only one broad and intense absorption band centered at $2.05 \mathrm{eV}$, with peaks of weaker intensities at lower energies, and questionable data in the range 2.25-2.95 eV. Comparing these experimental results with our calculations, for the planar isomer, peak II located at $1.96 \mathrm{eV}$, which is also the most intense peak of the calculated spectrum, is a candidate for the experimentally observed band center. Similarly, for the case of bipyramidal isomer, peaks III $(2.02 \mathrm{eV})$ and IV $(2.14 \mathrm{eV})$ are candidates for the observed band center. If indeed that is the case, then the calculated lower energy low-intensity peaks will correspond to lower energy part of the observed 
spectrum, which is also weaker in intensity. However, given the uncertainties associated with the experimental results on the photoabsorption spectrum of Na5 cluster,[40] we hope that in future more accurate measurements will be performed on this cluster, in order to facilitate a better comparison between experiment and theory.

As far as comparison of our computed spectra for the case of two isomers with the theoretical calculations of Kouteckỳ and coworkers[68] is concerned, there is good qualitative and quantitative agreement between two sets of calculations for the planar isomer. For example, the first major peak in the calculated spectrum of Koutecky and coworkers[68] for planar structure corresponds to two ${ }^{2} B_{2}$ type states located at 1.92 and $1.97 \mathrm{eV}$, while in our spectrum it corresponds to a single ${ }^{2} B_{2}$ type state, located at $1.96 \mathrm{eV}$.

Detailed information about the excited state wave functions, oscillator strengths, and photon polarizations corresponding to various optical transitions of the two isomers is presented in Tables 12 and 13 of the Supporting Information. On examining the excited state wave functions we find an interesting difference between the two isomers: for the planar structure, the wave functions of most of the optically active excited states are dominated by singly-excited configurations, while for the bipyramidal structure, they are dominated by double excitations.

Table 7: Comparison of location of peaks in the photoabsorption spectrum of $\mathrm{Na}_{5}$ cluster for different isomers, calculated using $6-311++\mathrm{G}(3 \mathrm{df}, 3 \mathrm{pd})$ basis set and optimized geometries, with experimental results. All photoabsorption calculations were performed using the frozen core QCI approach.

\begin{tabular}{|ccccccc|}
\hline \multirow{2}{*}{ Work } & \multirow{2}{*}{ Isomers } & \multicolumn{4}{c|}{ Peak Energies (Symmetry) (eV) } \\
\cline { 3 - 6 } & & I & II & III & IV & V \\
\hline This work & $C_{2 v}$-planar & $1.15\left({ }^{2} B_{1}\right)$ & $1.96\left({ }^{2} B_{2}\right)$ & $2.31\left({ }^{2} B_{1}\right)$ & $2.76\left({ }^{2} A_{1}\right)$ & \\
This work & $C_{2 v}$-pyramidal & $1.24\left({ }^{2} B_{1}\right)$ & $1.57\left({ }^{2} A_{1}\right)$ & $2.02\left({ }^{2} A_{1}\right)$ & $2.14\left({ }^{2} A_{1}\right)$ & $2.44\left({ }^{2} B_{1}\right)$ \\
Exp.(Ref.[40]) & - & 2.05 & & & & \\
\hline
\end{tabular}

\section{$3.2 .7 \mathrm{Na}_{6}$}

Here we have considered three different types of isomers with symmetries $C_{5 v}, D_{4 h}$, and planar $\left(D_{3 h}\right)$, as shown in Fig. 6. Out of these, we performed geometry optimization for $C_{5 v}$ and $D_{4 h}$ structures by employing the all-electron CCSD approach as implemented in the Psi4 code,[59] while for the planar $\left(D_{3 h}\right)$ isomer, we have used the geometry reported by Pal et at.[34] For the total energy calculations for all the three isomers, cc-PVDZ basis set was employed.[61] From Table 2 it is obvious that the ground state energies of all the three isomers are quite close to each other, with the $C_{5 v}$ capped pentagonal structure being the lowest in energy. Calculations for optical absorption spectra were performed using the frozen-core QCI approach employing 6-311++G(3df,3pd) basis set, and the results are presented in Fig. 16.

Before discussing our calculated spectra, we would like to briefly summarize the experimental results on the optical absorption of $\mathrm{Na}_{6}$ cluster as reported by Wang et al.[40] They reported four features in their measurement whose locations are reported in Table 8. As far as the measured intensity profile of the absorption spectrum is concerned, peak I was reported to be very weak, carrying lowest intensity compared 
to all other peaks, peak II was a very intense one with the highest intensity of the spectrum, peak III was also a very weak peak but with intensity higher than peak I, while peak IV was also intense, but with lower intensity as compared to peak II.[40] If we examine our calculated spectra presented in Figs. 16(a) and 16(b), we find that the computed spectra of $C_{5 v}$ and $D_{3 h}$ have exactly the same intensity pattern as observed in the experiment,[40] except that the location of peak I for the $D_{3 h}$ isomer is much smaller as compared to its experimental value. On the other hand, locations for all the peaks of the calculated spectra of $C_{5 v}$ and $D_{4 h}$ isomers are in very good agreement with the experiment, except that intensity profile of the $D_{4 h}$ does not match with the experimental one, because in the calculated spectrum peak III is the most intense one, as against peak II. Thus, considering both the intensity profile and the peak locations, we conclude that our calculated spectrum for the $C_{5 v}$ isomer is in the best agreement with the experiment. Thus, our conclusion disagrees with that of Wang et al.[40] who, based upon the theoretical calculations of Koutecky and coworkers,[68] concluded that the measured spectrum of the $\mathrm{Na}_{6}$ cluster was closest to that of the $D_{3 h}$ isomer. However, given the energetic proximity of all the three structures considered, it is certainly possible that the measured spectrum could be a mixed one, carrying features of the absorption spectra of all the isomers.

Wave functions, along with the polarization characteristics of the excited states contributing to the peaks in the calculated absorption spectra of $C_{5 v}, D_{3 h}$, and $D_{4 h}$ isomers are presented in Tables 14, 15, and 16 of the Supporting Information. Upon examination of these tables, following common features emerge: (a) all the isomers have doubly-degenerate HOMO orbitals, (b) first three peaks in the spectra for all the three isomers correspond to transitions polarized "in-plane", while the fourth peak corresponds to "perpendicular" polarization, and (c) wave functions of the excited states contributing to these peaks for all the three isomers exhibit strong mixing of singlyexcited configurations with almost similar weights. According to a criterion proposed by Kouteckỳ and coworkers,[69] excited states whose wave functions are a mixture of a large number of excited particle-hole configurations with almost identical weights, signify the emergence of collective plasmonic excitations. This suggests that the optical excitations in $\mathrm{Na}_{6}$ cluster are plasmonic in nature, and, therefore, we investigate this possibility further. Deshpande et al. computed the optical absorption spectra of $\mathrm{Na}_{8-x} \mathrm{Li}_{x},[70]$ and bare aluminum clusters,[71] using first principles time-dependent local-density approximation (TDLDA). For the case of $\mathrm{Al}_{n}$ clusters they concluded that for $n \geq 6$, presence of broad absorption bands corresponds to the emergence of surface plasma resonances, with the increasing size.[71] For the case of $\mathrm{Na}_{8}{ }_{x} \mathrm{Li}_{x}$ clusters, authors identified the most intense peak with the plasmon resonance,[70] which, for the case of $\mathrm{Na}_{8}$ clusters, is located at $\approx 2.7 \mathrm{eV}$. [70] If we compare our computed absorption spectra of various $\mathrm{Na}_{6}$ isomers ( $c f$. Fig. 16), with that of $\mathrm{Na}_{8}$ cluster reported by Deshpande et al.[70], we observe the following trends: (a) both $\mathrm{Na}_{6}$ and $\mathrm{Na}_{8}$ spectra are dominated by one high intensity peak, accompanied by several peaks of weaker intensities, (b) relative intensities of the weaker peaks for $\mathrm{Na}_{6}$ is significantly larger than those for $\mathrm{Na}_{8}$. If we compare the peak locations of the most intense peaks for these clusters, for $\mathrm{Na}_{6}$ they are located at $1.94 \mathrm{eV}\left(D_{3 h}\right), 2.15 \mathrm{eV}\left(C_{5 v}\right), 2.34 \mathrm{eV}\left(D_{4 h}\right)$, while for $\mathrm{Na}_{8}$ it is located at $2.70 \mathrm{eV}$. Thus, most intense peaks in the absorption spectra of $\mathrm{Na}_{6}$ isomers are at significantly lower energies as compared to that for $\mathrm{Na}_{8}$. However, if we allow for the possibility that the higher energy, lower intensity, peaks could also be due to plasmonic excitations, then we note that locations of peak IV in the spectra of the three isomers of $\mathrm{Na}_{6}$ at $2.81 \mathrm{eV}\left(D_{3 h}\right), 2.79 \mathrm{eV}\left(C_{5 v}\right), 2.71 \mathrm{eV}\left(D_{4 h}\right)$, are rela- 
tively independent of their geometrical structure, and also in good agreement with the plasmonic peak of $\mathrm{Na}_{8}$ at $2.70 \mathrm{eV}$. Furthermore, the excited state wave functions of these peaks presented in the Supporting Information satisfy the configuration mixing criterion of Koutecky and coworkers,[69] mentioned above. To resolve this uncertainty regarding the location of surface plasmonic resonances in Na clusters, it will be useful to perform such calculations in larger clusters starting with $\mathrm{Na}_{8}$.

Table 8: Comparison of location of peaks in the photoabsorption spectrum of $\mathrm{Na}_{6}$ cluster for different isomers, calculated using $6-311++\mathrm{G}(3 \mathrm{df}, 3 \mathrm{pd})$ basis set and optimized geometries, with experimental results. All photoabsorption calculations were performed using the frozen core QCI approach.

\begin{tabular}{|ccccccc|}
\hline \multirow{2}{*}{ Work } & \multirow{2}{*}{ Method } & \multirow{2}{*}{ Isomers } & \multicolumn{4}{c|}{ Peak Energies (Symmetry) (eV) } \\
\cline { 4 - 7 } & & & I & II & III & IV \\
\hline This work & QCI & $D_{4 h}$ & $1.65\left({ }^{1} E_{u}\right)$ & $1.90\left({ }^{1} E_{u}\right)$ & $2.34\left({ }^{1} E_{u}\right)$ & $2.79\left({ }^{1} A_{2 u}\right)$ \\
This work & QCI & $D_{3 h}$ & $1.36\left({ }^{1} E^{\prime}\right)$ & $1.94\left({ }^{1} E^{\prime}\right)$ & $2.49\left({ }^{1} E^{\prime}\right)$ & $2.80\left({ }^{1} A_{2}^{\prime \prime}\right)$ \\
This work & QCI & $C_{5 v}$ & $1.19\left({ }^{1} E_{1}\right)$ & $2.15\left({ }^{1} E_{1}\right)$ & $2.52\left({ }^{1} E_{1}\right)$ & $2.79\left({ }^{1} A_{1}\right)$ \\
Exp.(Ref.[40]) & - & - & 1.78 & 2.08 & 2.44 & 2.83 \\
\hline
\end{tabular}

\section{CONCLUSION}

In this work we presented ab initio calculations of electronic structure and optical properties of small sodium clusters, utilizing configuration-interaction methodology based upon Gaussian type of basis functions. Several isomers of clusters ranging from $\mathrm{Na}_{2}$ to $\mathrm{Na}_{6}$ were considered, and the calculated spectra were compared with the experimental results, where available. In most cases, we obtained very good agreement with the experiments, thus validating our methodology. We also analyzed the wave functions of the excited states corresponding to various resonances in the spectra, and found them to be almost equal mixtures of several configurations suggesting the possibility that those states correspond to collective plasmonic excitations.[68] In light of the TDLDA calculations of optical absorption spectrum of Nas cluster by Deshpande et al.[70] reporting a plasmon peak located around $2.7 \mathrm{eV}$, in future we intend to perform similar calculations of optical absorption spectra of medium sized Na clusters starting with $\mathrm{Na}_{8}$ aimed at exploring the location and nature of plasmons in these systems.

As far as geometry optimization of various clusters is concerned, we found that core excitations play an important role, and, therefore, all-electron correlated calculations are essential. But, all the photoabsorption calculations were performed using the frozen-core approximation, therefore, good agreement between our results, and the experiments, indicates that the models of sodium clusters assuming a single valence electron per atom, are well founded, when it comes to low-lying excitations. In future, we propose to perform similar studies on clusters composed of other metal atoms such as lithium and berylium.

Acknowledgements We gratefully acknowledge the financial support of Department of Science and Technology (DST), India, under the grant \# SB/S2/CMP-066/2013. PKP acknowledges the paid leave granted to him to pursue his Ph.D. under the Faculty Improvement Program of University Grants Commission (UGC), India. 


\section{Author Contribution Statement}

A.S. conceptualized the problem, explained the calculation procedure to P.K.P. and D.K.R, and wrote the paper. P.K.P. and D.K.R. performed the calculations, and analyzed the generated data.

\section{References}

1. Y. Kawazoe, T. Kondow, K. Ohno (eds.), Clusters and Nanomaterials Theory and Experiment (Springer, Berlin, 2002)

2. J.A. Alonso, Structure and properties of atomic nanoclusters (Imperial Coll., London, 2005)

3. J. Jellinek (ed.), Theory of Atomic and Molecular Clusters With a Glimpse at Experiments (Springer, Berlin, 1999)

4. W.A. de Heer, Reviews of Modern Physics 65, 611 (1993). DOI 10.1103/RevModPhys.65. 611

5. W. Ekardt (ed.), Metal Clusters (Wiley, New York, 1999)

6. J. Bowlan, A. Liang, W.A. de Heer, Phys. Rev. Lett. 106, 043401 (2011). DOI 10. 1103/PhysRevLett.106.043401. URL http://link.aps.org/doi/10.1103/PhysRevLett. 106.043401

7. M. Brack, Rev. Mod. Phys. 65, 677 (1993). DOI 10.1103/RevModPhys.65.677. URL http://link.aps.org/doi/10.1103/RevModPhys.65.677

8. C. Wang, S. Pollack, M.M. Kappes, Chemical Physics Letters 166(1), 26 (1990). DOI http://dx.doi.org/10.1016/0009-2614(90)87044-R. URL http://www.sciencedirect.com/ science/article/pii/000926149087044R

9. K. Clemenger, Phys. Rev. B 32, 1359 (1985). DOI 10.1103/PhysRevB.32.1359. URL http://link.aps.org/doi/10.1103/PhysRevB.32.1359

10. D.E. Beck, Phys. Rev. B 30, 6935 (1984). DOI 10.1103/PhysRevB.30.6935. URL http: //link.aps.org/doi/10.1103/PhysRevB.30.6935

11. W. Ekardt, Phys. Rev. Lett. 52, 1925 (1984). DOI 10.1103/PhysRevLett.52.1925. URL http://link.aps.org/doi/10.1103/PhysRevLett.52.1925

12. C. Yannouleas, R.A. Broglia, M. Brack, P.F. Bortignon, Phys. Rev. Lett. 63, 255 (1989). DOI 10.1103/PhysRevLett.63.255. URL http://link.aps.org/doi/10.1103/ PhysRevLett.63.255

13. V. Bonačić-Kouteckỳ, J. Gaus, M. Guest, L. Čšpiva, J. Koutecký, Chemical Physics Letters 206(5-6), 528 (1993). DOI http://dx.doi.org/10.1016/0009-2614(93)80179-S. URL http://www.sciencedirect.com/science/article/pii/000926149380179S

14. B.K. Rao, P. Jena, Phys. Rev. B 32, 2058 (1985). DOI 10.1103/PhysRevB.32.2058. URL http://link.aps.org/doi/10.1103/PhysRevB.32.2058

15. J.L. Martins, J. Buttet, R. Car, Phys. Rev. B 31, 1804 (1985). DOI 10.1103/PhysRevB. 31.1804. URL http://link.aps.org/doi/10.1103/PhysRevB.31.1804

16. I. Boustani, W. Pewestorf, P. Fantucci, V. Bonačić-Kouteckỳ, J. Koutecký, Phys. Rev. B 35, 9437 (1987). DOI 10.1103/PhysRevB.35.9437. URL http://link.aps.org/doi/10. 1103/PhysRevB.35.9437

17. V. Bonačić-Kouteckỳ, P. Fantucci, J. Koutecký, Phys. Rev. B 37, 4369 (1988). DOI 10.1103/PhysRevB.37.4369. URL http://link.aps.org/doi/10.1103/PhysRevB.37.4369

18. B.K. Rao, P. Jena, Phys. Rev. B 37, 2867 (1988). DOI 10.1103/PhysRevB.37.2867. URL http://link.aps.org/doi/10.1103/PhysRevB.37.2867

19. V. Bonačić-Kouteckỳ, P. Fantucci, J. Kouteckỳ, The Journal of Chemical Physics 91(6), $3794(1989)$

20. V. Bonačić-Kouteckỳ, P. Fantucci, J. Koutecký, Chemical Reviews 91(5), 1035 (1991). DOI 10.1021/cr00005a016. URL http://pubs.acs.org/doi/abs/10.1021/cr00005a016

21. V. Bonačić-Kouteckỳ, J. Pittner, C. Fuchs, P. Fantucci, M. Guest, J. Kouteckỳ, The Journal of chemical physics 104(4), 1427 (1996)

22. V. Bonačić-Kouteckỳ, P. Fantucci, J. Kouteckỳ, Chemical Physics Letters 166(1), 32 (1990). DOI http://dx.doi.org/10.1016/0009-2614(90)87045-S. URL http://www . sciencedirect.com/science/article/pii/000926149087045S 
23. U. Röthlisberger, W. Andreoni, The Journal of Chemical Physics 94(12), 8129 (1991). DOI http://dx.doi.org/10.1063/1.460096. URL http://scitation.aip.org/content/ aip/journal/jcp/94/12/10.1063/1.460096

24. S.A. Blundell, C. Guet, R.R. Zope, Phys. Rev. Lett. 84, 4826 (2000). DOI 10.1103/ PhysRevLett.84.4826. URL http://link.aps.org/doi/10.1103/PhysRevLett.84.4826

25. M.B. Torres, L.C. Balbás, J.of Physics: Condensed Matter 14(23), 5795 (2002). URL http: //stacks.iop.org/0953-8984/14/i=23/a=311

26. I.A. Solov'yov, A.V. Solov'yov, W. Greiner, Phys. Rev. A 65, 053203 (2002). DOI 10.1103/ PhysRevA.65.053203. URL http://link.aps.org/doi/10.1103/PhysRevA.65.053203

27. S. Kümmel, M. Brack, P.G. Reinhard, Phys. Rev. B 62, 7602 (2000). DOI 10.1103/ PhysRevB.62.7602. URL http://link.aps.org/doi/10.1103/PhysRevB.62.7602

28. J.M. Pacheco, J.L. Martins, The Journal of Chemical Physics 106(14), 6039 (1997). DOI http://dx.doi.org/10.1063/1.473608. URL http://scitation.aip.org/content/ aip/journal/jcp/106/14/10.1063/1.473608

29. M. Moseler, H. Häkkinen, U. Landman, Phys. Rev. Lett. 87, 053401 (2001). DOI 10.1103/PhysRevLett.87.053401. URL http://link.aps.org/doi/10.1103/PhysRevLett. 87.053401

30. A. Rubio, J.A. Alonso, X. Blase, L.C. Balbás, S.G. Louie, Phys. Rev. Lett. 77, 247 (1996). DOI 10.1103/PhysRevLett.77.247. URL http://link.aps.org/doi/10.1103/ PhysRevLett.77.247

31. M.A.L. Marques, A. Castro, A. Rubio, The Journal of Chemical Physics 115(7), 3006 (2001). DOI http://dx.doi.org/10.1063/1.1385368. URL http://scitation.aip.org/ content/aip/journal/jcp/115/7/10.1063/1.1385368

32. J.O. Joswig, L.O. Tunturivuori, R.M. Nieminen, The Journal of Chemical Physics 128(1), 014707 (2008). DOI http://dx.doi.org/10.1063/1.2814161. URL http://scitation.aip. org/content/aip/journal/jcp/128/1/10.1063/1.2814161

33. B.J. Wang, Y. Xu, S.H. Ke, The Journal of Chemical Physics 137(5), 054101 (2012). DOI http://dx.doi.org/10.1063/1.4739952. URL http://scitation.aip.org/content/ aip/journal/jcp/137/5/10.1063/1.4739952

34. G. Pal, G. Lefkidis, H.C. Schneider, W. Hübner, The Journal of Chemical Physics 133(15), 154309 (2010). DOI http://dx.doi.org/10.1063/1.3494093. URL http://scitation.aip. org/content/aip/journal/jcp/133/15/10.1063/1.3494093

35. G. Pal, Y. Pavlyukh, W. HÃACbner, H.C. Schneider, The European Physical Journal B 79(3), 327 (2011). DOI 10.1140/epjb/e2010-10033-1. URL http://dx.doi.org/10.1140/ epjb/e2010-10033-1

36. W.R. Fredrickson, W.W. Watson, Phys. Rev. 30, 429 (1927). DOI 10.1103/PhysRev.30. 429. URL http://link.aps.org/doi/10.1103/PhysRev.30.429

37. A. Herrmann, M. Hofmann, S. Leutwyler, E. Schumacher, L. Wöste, Chemical Physics Letters 62(2), 216 (1979). DOI http://dx.doi.org/10.1016/0009-2614(79)80162-1. URL http://www.sciencedirect.com/science/article/pii/0009261479801621

38. C.R. Chris Wang, S. Pollack, D. Cameron, M.M. Kappes, The Journal of Chemical Physics 93(6), 3787 (1990). DOI http://dx.doi.org/10.1063/1.458765. URL http://scitation. aip.org/content/aip/journal/jcp/93/6/10.1063/1.458765

39. M. Broyer, G. Delacrétaz, P. Labastie, R. Whetten, J. Wolf, L. Wöste, Zeitschrift für Physik D Atoms, Molecules and Clusters 3(2), 131 (1986). DOI 10.1007/BF01384797. URL http://dx.doi.org/10.1007/BF01384797

40. C.R.C. Wang, S. Pollack, T.A. Dahlseid, G.M. Koretsky, M.M. Kappes, The Journal of Chemical Physics 96(11), 7931 (1992). DOI http://dx.doi.org/10.1063/1.462345. URL http://scitation.aip.org/content/aip/journal/jcp/96/11/10.1063/1.462345

41. M. Schmidt, H. Haberland, The European Physical Journal D - Atomic, Molecular, Optical and Plasma Physics 6(1), 109 (1999). DOI 10.1007/s100530050290. URL http://dx.doi. org/10.1007/s100530050290

42. W.D. Knight, K. Clemenger, W.A. de Heer, W.A. Saunders, Phys. Rev. B 31, 2539 (1985). DOI 10.1103/PhysRevB.31.2539. URL http://link.aps.org/doi/10.1103/PhysRevB.31. 2539

43. W.A. de Heer, K. Selby, V. Kresin, J. Masui, M. Vollmer, A. Chatelain, W.D. Knight, Phys. Rev. Lett. 59, 1805 (1987). DOI 10.1103/PhysRevLett.59.1805. URL http://link. aps.org/doi/10.1103/PhysRevLett.59.1805

44. K. Selby, M. Vollmer, J. Masui, V. Kresin, W.A. de Heer, W.D. Knight, Phys. Rev. B 40, 5417 (1989). DOI 10.1103/PhysRevB.40.5417. URL http://link.aps.org/doi/10.1103/ PhysRevB. 40.5417 
45. K. Selby, V. Kresin, J. Masui, M. Vollmer, W.A. de Heer, A. Scheidemann, W.D. Knight, Phys. Rev. B 43, 4565 (1991). DOI 10.1103/PhysRevB.43.4565. URL http://link.aps . org/doi/10.1103/PhysRevB.43.4565

46. M.E. Casida, Journal of Molecular Structure: \{THEOCHEM\} 914(1-3), 3 (2009). DOI http://dx.doi.org/10.1016/j.theochem.2009.08.018. URL http://www.sciencedirect. com/science/article/pii/S0166128009005363. Time-dependent density-functional theory for molecules and molecular solids

47. A. Shukla, Phys. Rev. B 65, 125204 (2002). DOI 10.1103/PhysRevB.65.125204. URL http://link.aps.org/doi/10.1103/PhysRevB.65.125204

48. A. Shukla, Chem. Phys. 300(13), 177 (2004). DOI http://dx.doi.org/10.1016/ j.chemphys.2004.02.004. URL http://www.sciencedirect.com/science/article/pii/ S030101040400076X

49. A. Shukla, Phys. Rev. B 69, 165218 (2004). DOI 10.1103/PhysRevB.69.165218. URL http://link.aps.org/doi/10.1103/PhysRevB.69.165218

50. P. Sony, A. Shukla, Phys. Rev. B 75, 155208 (2007). DOI 10.1103/PhysRevB.75.155208. URL http://link.aps.org/doi/10.1103/PhysRevB.75.155208

51. P. Sony, A. Shukla, Phys. Rev. B 71, 165204 (2005). DOI 10.1103/PhysRevB.71.165204. URL http://link.aps.org/doi/10.1103/PhysRevB.71.165204

52. H. Chakraborty, A. Shukla, The Journal of Physical Chemistry A 117(51), 14220 (2013). DOI 10.1021/jp408535u. URL http://pubs.acs.org/doi/abs/10.1021/jp408535u

53. H. Chakraborty, A. Shukla, The Journal of Chemical Physics 141(16), 164301 (2014). DOI http://dx.doi.org/10.1063/1.4897955. URL http://scitation.aip.org/content/ aip/journal/jcp/141/16/10.1063/1.4897955

54. S. Sahu, A. Shukla, Nanoscale Research Letters 5(4), 714 (2010). DOI 10.1007/ s11671-010-9536-y. URL http://dx.doi.org/10.1007/s11671-010-9536-y

55. R. Shinde, A. Shukla, Nano Life 2(02), 1240004 (2012). DOI 10.1142/S1793984411000529. URL http://www. worldscientific.com/doi/abs/10.1142/S1793984411000529

56. R. Shinde, A. Shukla, Phys. Chem. Chem. Phys. 16, 20714 (2014). DOI 10.1039/ C4CP02232G. URL http://dx.doi.org/10.1039/C4CP02232G

57. R. Shinde, A. Shukla, The European Physical Journal D 67(5), 98 (2013). DOI 10.1140/ epjd/e2013-30546-3. URL http://dx.doi.org/10.1140/epjd/e2013-30546-3

58. L.E. McMurchie, S.T. Elbert, S.R. Langhoff, E.R. Davidson. MELD package from Indiana University. It has been modified by us to handle bigger systems.

59. J.M. Turney, A.C. Simmonett, R.M. Parrish, E.G. Hohenstein, F. Evangelista, J.T. Fermann, B.J. Mintz, L.A. Burns, J.J. Wilke, M.L. Abrams, N.J. Russ, M.L. Leininger, C.L. Janssen, E.T. Seidl, W.D. Allen, H.F. Schaefer, R.A. King, E.F. Valeev, C.D. Sherrill, T.D. Crawford, WIREs Comput. Mol. Sci. 2, 556 (2012). DOI http://dx.doi.org/10.1002/ wcms.93

60. K.L. Schuchardt, B.T. Didier, T. Elsethagen, L. Sun, V. Gurumoorthi, J. Chase, J. Li, T.L. Windus, Journal of Chemical Information and Modeling 47(3), 1045 (2007). DOI 10.1021/ ci600510j. URL http://pubs.acs.org/doi/abs/10.1021/ci600510j. PMID: 17428029

61. D. Feller, Journal of Computational Chemistry 17(13), 1571 (1996). DOI 10.1002/(SICI) 1096-987X(199610)17:13<1571::AID-JCC9>3.0.CO;2-P. URL http://dx.doi.org/10. 1002/ (SICI) 1096-987X (199610) $17: 13<1571:$ : AID- JCC9>3 . 0. CO; 2-P

62. T. Ozaki, H. Kino, Phys. Rev. B 69, 195113 (2004). DOI 10.1103/PhysRevB.69.195113. URL http://link.aps.org/doi/10.1103/PhysRevB.69.195113

63. P. Calaminici, K. Jug, A.M. Köster, The Journal of chemical physics 111(10), 4613 (1999)

64. V. Bonačić-Kouteckỳ, P. Fantucci, J. Kouteckỳ, The Journal of Chemical Physics 93(6), 3802 (1990). DOI http://dx.doi.org/10.1063/1.458766. URL http://scitation.aip.org/ content/aip/journal/jcp/93/6/10.1063/1.458766

65. M. Schmidt, C. Ellert, W. Kronmüller, H. Haberland, Phys. Rev. B 59, 10970 (1999). DOI 10.1103/PhysRevB.59.10970. URL http://link.aps.org/doi/10.1103/PhysRevB. 59. 10970

66. C. Ellert, M. Schmidt, T. Reiners, H. Haberland, Zeitschrift für Physik D Atoms, Molecules and Clusters 39(4), 317 (1997). DOI 10.1007/s004600050142. URL http://dx.doi.org/ $10.1007 / \mathrm{s} 004600050142$

67. V. Bonaić-Kouteckỳ, P. Fantucci, J. Kouteckỳ, Physical Review B 37(9), 4369 (1988)

68. Bonačić-Kouteckỳ, V. and Pittner, J. and Scheuch, C. and Guest, M. F. and Koutecký, J., The Journal of Chemical Physics 96(11), 7938 (1992). DOI http://dx.doi.org/10.1063/1. 462346. URL http://scitation.aip.org/content/aip/journal/jcp/96/11/10.1063/1. 462346 
69. J. Blanc, V.B. Koutecký, M. Broyer, J. Chevaleyre, P. Dugourd, J. Koutecký, C. Scheuch, J.P. Wolf, L. Wöste, J. Chem. Phys. 96(3), 1793 (1992). DOI DOI:10.1063/1.462846. URL http://dx.doi.org/10.1063/1.462846

70. M.D. Deshpande, D.G. Kanhere, P.V. Panat, I. Vasiliev, R.M. Martin, Phys. Rev. A 65, 053204 (2002). DOI 10.1103/PhysRevA.65.053204. URL http://link.aps.org/doi/10. 1103/PhysRevA.65.053204

71. M.D. Deshpande, D.G. Kanhere, I. Vasiliev, R.M. Martin, Phys. Rev. B 68, 035428 (2003). DOI 10.1103/PhysRevB.68.035428. URL http://link.aps.org/doi/10.1103/PhysRevB. 68.035428 


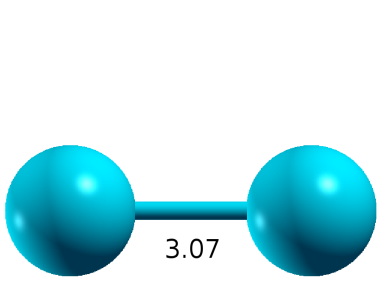

(a) $\mathrm{Na}_{2}, \mathrm{D}_{\infty h}$

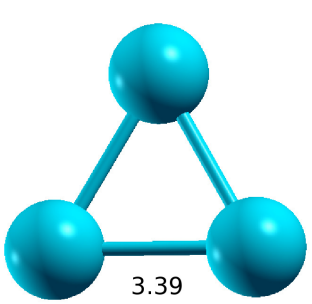

(b) $\mathrm{Na}_{3}^{+}, \mathrm{D}_{3 h}$

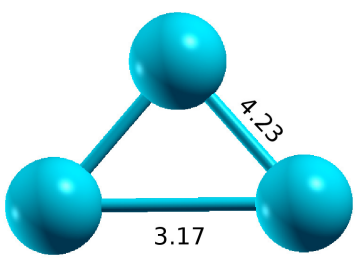

(c) $\mathrm{Na}_{3}, \mathrm{C}_{2 v}$

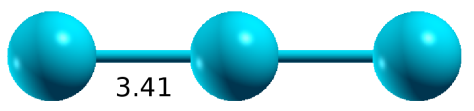

(d) $\mathrm{Na}_{3}, \mathrm{D}_{\infty h}$

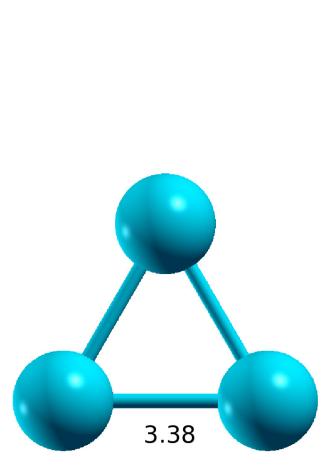

(e) $\mathrm{Na}_{3}, \mathrm{D}_{3 h}$

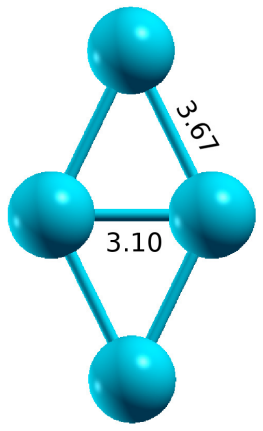

(f) $\mathrm{Na}_{4}, \mathrm{D}_{2 h}$

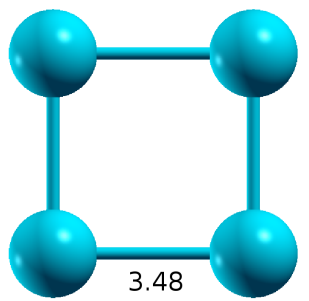

(g) $\mathrm{Na}_{4}, \mathrm{D}_{4 h}$

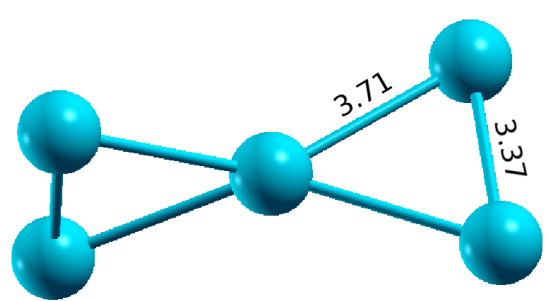

(h) $\mathrm{Na}_{5}^{+}, \mathrm{D}_{2 d}$

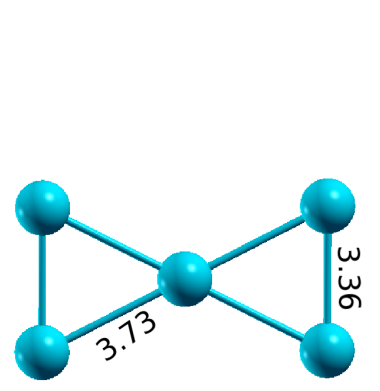

(i) $\mathrm{Na}_{5}^{+}, \mathrm{D}_{2 h}$

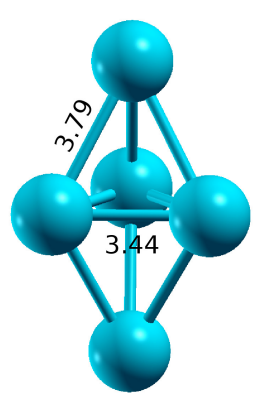

(j) $\mathrm{Na}_{5}^{+}, \mathrm{D}_{3 h}$

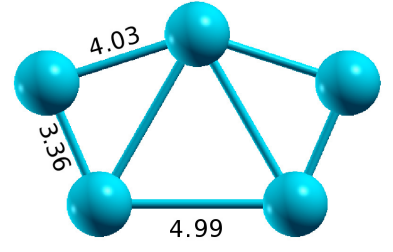

(k) $\mathrm{Na}_{5}^{+}, \mathrm{C}_{2 v}$

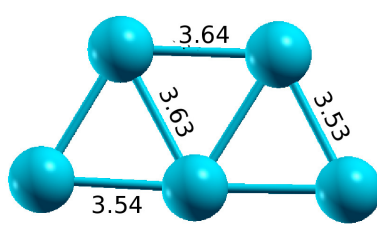

(l) $\mathrm{Na}_{5}, \mathrm{C}_{2 v}-$ planar

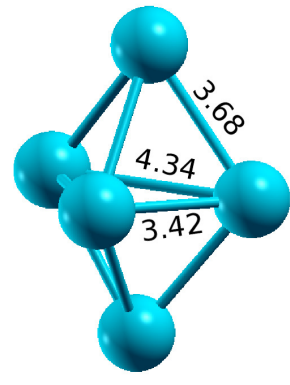

(m) $\mathrm{Na}_{5}, \mathrm{C}_{2 v}-$ bipyramidal

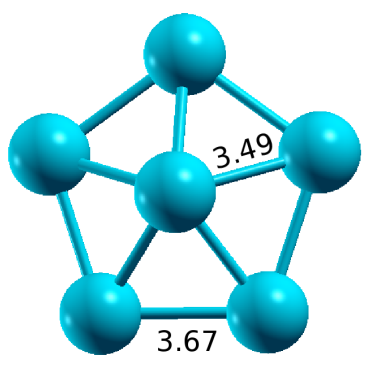

(n) $\mathrm{Na}_{6}, \mathrm{C}_{5 v}$

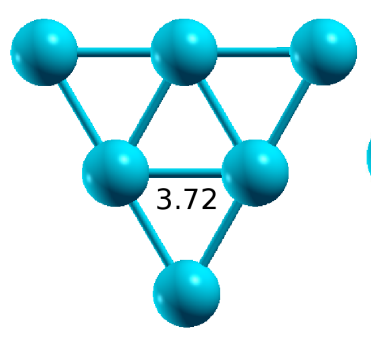

(o) $\mathrm{Na}_{6}, \mathrm{D}_{3 h}$

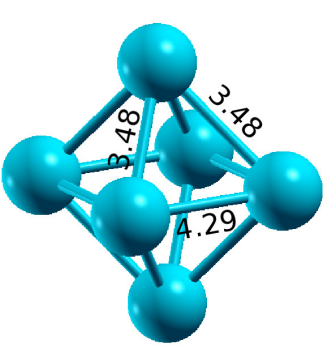

(p) $\mathrm{Na}_{6}, \mathrm{D}_{4 h}$

Fig. 6: Ground state geometries of different sodium clusters considered in this work, with their bond lengths $(\AA)$ indicated in the figures. 


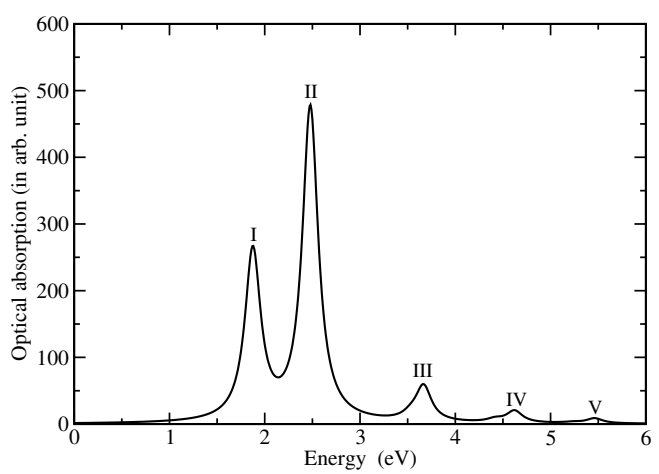

Fig. 7: Optical absorption spectrum of $\mathrm{Na}_{2}$ cluster computed using the frozen core FCI approach. For plotting the spectrum, a uniform linewidth of $0.1 \mathrm{eV}$ was used.

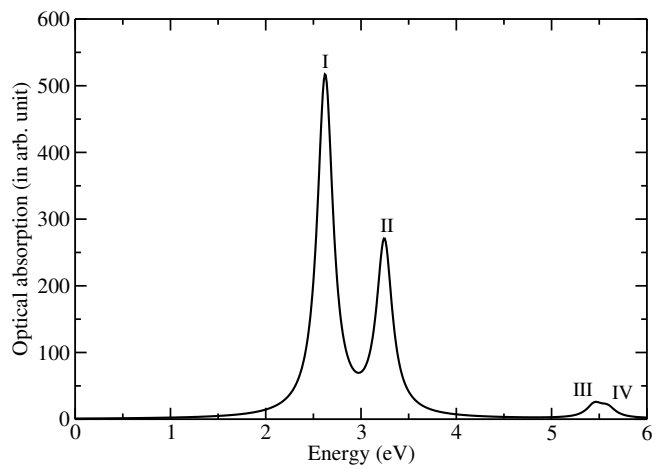

Fig. 8: Optical absorption spectrum of $\mathrm{Na}_{3}{ }^{+}$cluster computed using the frozen core full CI approach, and $6-311++\mathrm{G}(3 \mathrm{df}, 3 \mathrm{pd})$ basis set. For plotting the spectrum, a uniform linewidth of $0.1 \mathrm{eV}$ was used. 


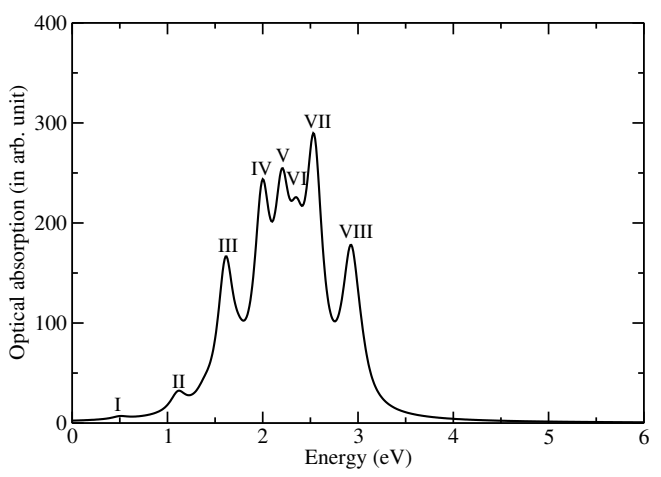

Fig. 9: Optical absorption spectrum of $\mathrm{Na}_{3}$ isosceles triangular cluster computed using the frozen core full CI approach, and $6-311++\mathrm{G}(3 \mathrm{df}, 3 \mathrm{pd})$ basis set. For plotting the spectrum, a uniform line width of $0.1 \mathrm{eV}$ was used.

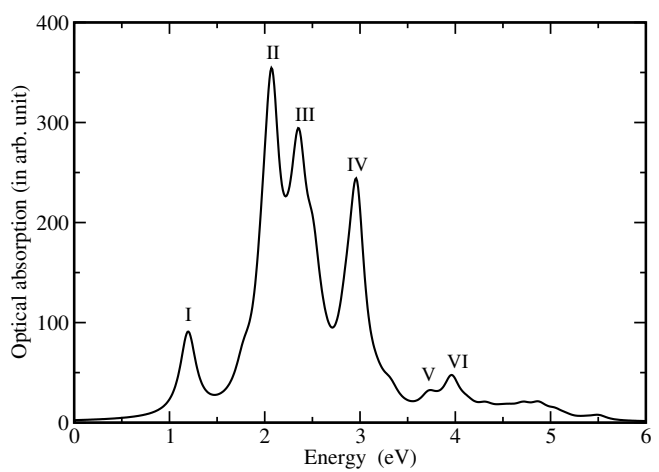

Fig. 10: Optical absorption spectrum of $\mathrm{Na}_{3}$ - equilateral atomic cluster with frozen core FCI. For plotting the spectrum, a uniform linewidth of $0.1 \mathrm{eV}$ was used. 


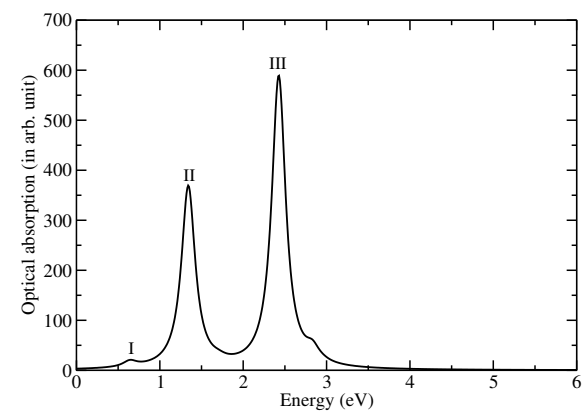

Fig. 11: Optical absorption spectrum of linear isomer of $\mathrm{Na}_{3}$ cluster, computed using the frozen core FCI approach. For plotting the spectrum, a uniform linewidth of 0.1 $\mathrm{eV}$ was used.

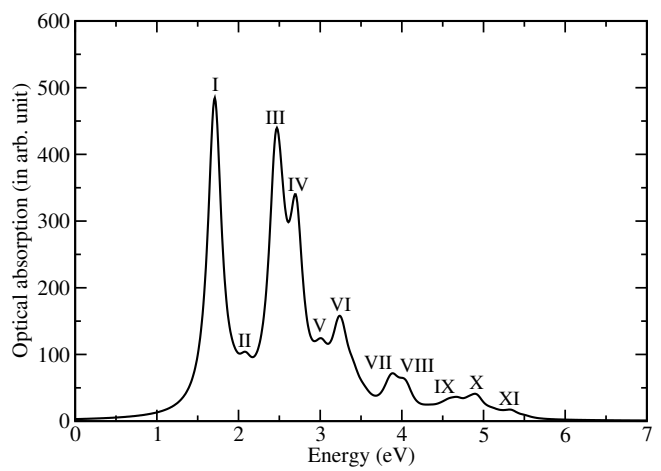

Fig. 12: Optical absorption spectrum of rhombus shaped $\mathrm{Na}_{4}$ cluster, performed using the frozen-core FCI approach. For plotting the spectrum, a uniform line width of 0.1 $\mathrm{eV}$ was used. 


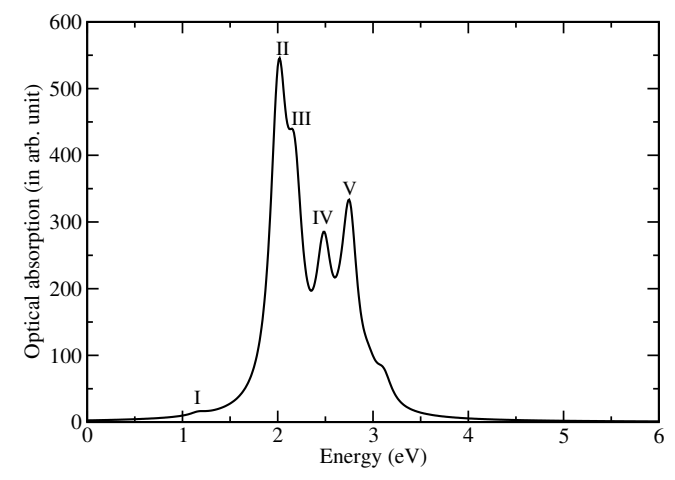

Fig. 13: Optical absorption spectrum of $\mathrm{Na}_{4}$ - square-shaped atomic cluster computed using the frozen core FCI method. For plotting the spectrum, a uniform linewidth of $0.1 \mathrm{eV}$ was used.
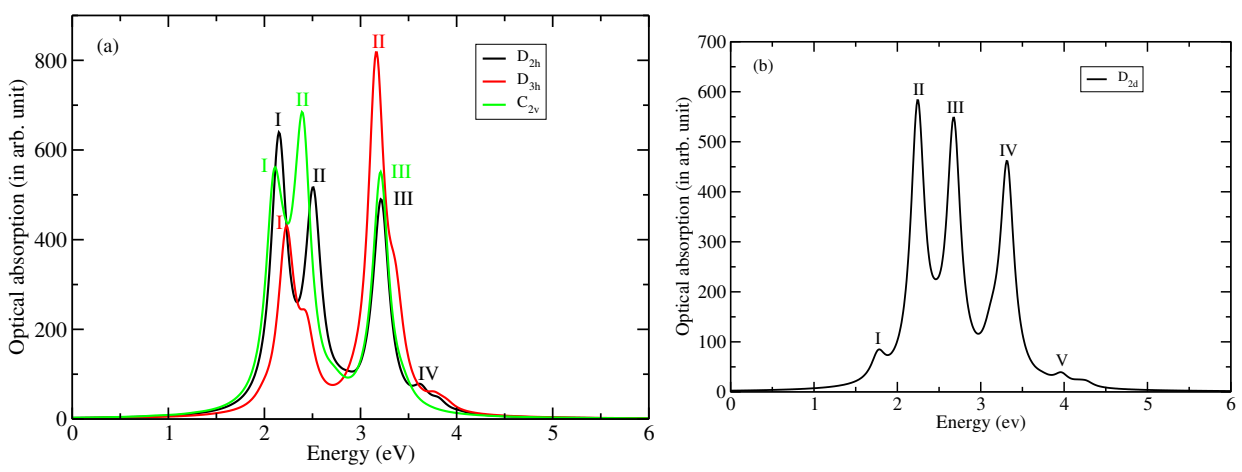

Fig. 14: Optical absorption spectra of various $\mathrm{Na}_{5}{ }^{+}$isomers with: (a) $D_{2 h}, D_{3 h}$, and $C_{2 v}$ symmetries, and (b) $D_{2 d}$ symmetry. These spectra were computed using the frozen core FCI approach, employing a $6-311++\mathrm{G}(3 \mathrm{df}, 3 \mathrm{pd})$ basis set. For plotting the spectra, a uniform linewidth of $0.1 \mathrm{eV}$ was used. 

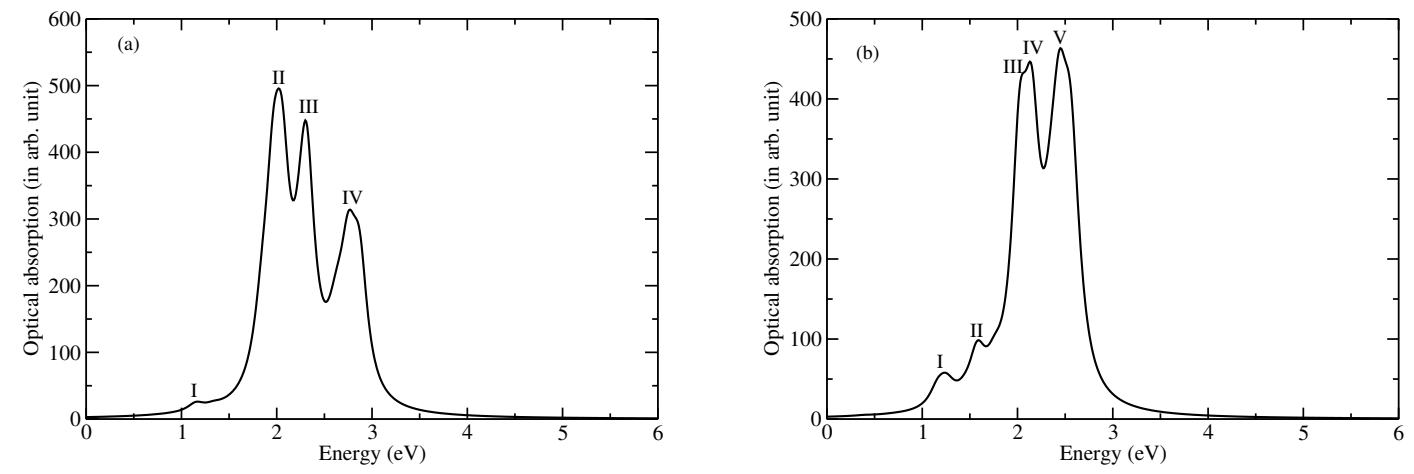

Fig. 15: Optical absorption spectra of $\mathrm{Na}_{5}$ isomers with: (a) $C_{2 v}$ planar structure, and (b) $C_{2 v}$ trigonal bipyramidal structure. These spectra were computed using the frozen core QCI approach, employing a $6-311++\mathrm{G}(3 \mathrm{df}, 3 \mathrm{pd})$ basis set. For plotting the spectra, a uniform linewidth of $0.1 \mathrm{eV}$ was used.
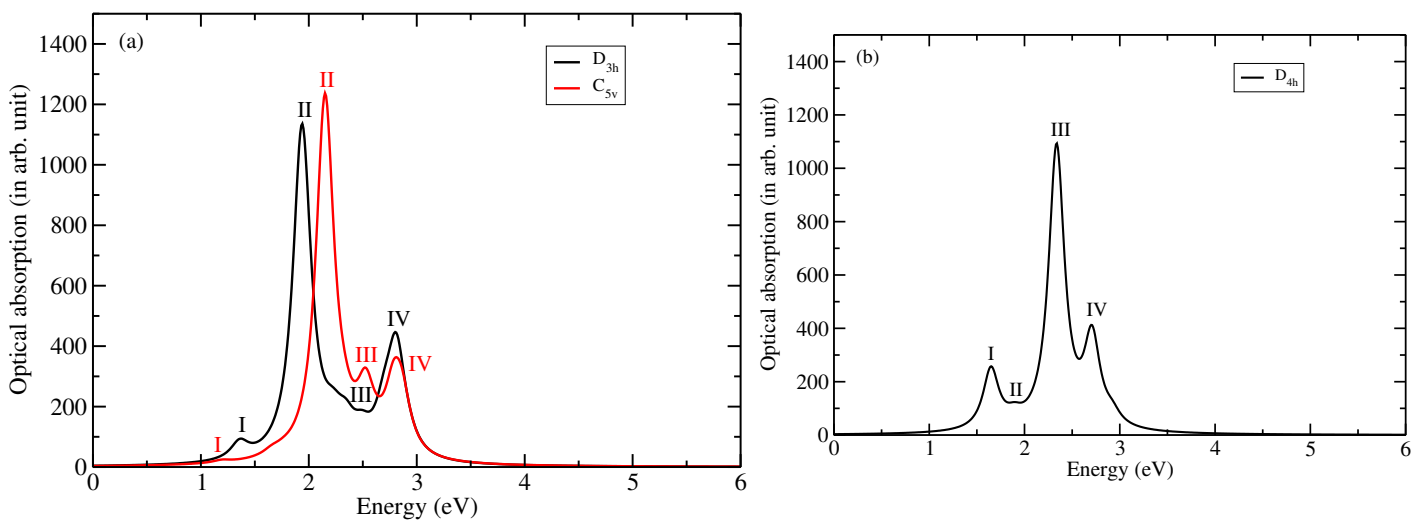

Fig. 16: Optical absorption spectra of various $\mathrm{Na}_{6}$ isomers with: (a) $D_{3 h}$ and $C_{5 v}$ symmetries, and (b) $D_{4 h}$ symmetry. These spectra were computed using the frozen core QCI approach, employing a 6-311++G(3df,3pd) basis set. For plotting the spectra, a uniform linewidth of $0.1 \mathrm{eV}$ was used. 


\title{
Supporting Information for Photoabsorption
} in Sodium Clusters: First Principles Configuration Interaction Calculations

\author{
Pradip Kumar Priya, Deepak Kumar Rai, and Alok Shukla* \\ Department of Physics, Indian Institute of Technology Bombay, Powai, Mumbai 400076, \\ INDIA \\ E-mail: emails:eccpradip@gmail.com, deepakrai@phy.iitb.ac.in,shukla@phy.iitb.ac.in
}

\section{Excited State Wave Functions, Energies, and Transition Moments}

In the following tables we present the excitation energies, the many-particle wave functions, and the transition dipole moments with respect to the ground state, of the excited states corresponding to the peaks in the linear absorption spectrum of $\mathrm{Na}$ atomic clusters, discussed in the main text. 
Table 1: Excitation energies, E, and many-particle wave functions of excited states corresponding to the peaks in the linear absorption spectrum of $\mathrm{Na}_{2}$ ( $c f$. Fig. 7, in main text), along with the oscillator strength $f$ of the transitions. Polarization corresponding to the transition is denoted as $\|$, if it is along the molecule, and $\perp$, if it is perpendicular to it. In the wave function, the bracketed numbers are the CI coefficients of a given electronic configuration, and symbols $H / L$ denote $\mathrm{HOMO} / \mathrm{LUMO}$ orbitals.

\begin{tabular}{|c|c|c|c|c|c|}
\hline Peak & $E(\mathrm{eV})$ & Symmetry & $f$ & Polarization & Wave Function \\
\hline \multirow[t]{4}{*}{ I } & 1.87 & ${ }^{1} \Sigma_{u}$ & 0.6241 & & $|H \rightarrow L\rangle(0.5829)$ \\
\hline & & & & & $|H \rightarrow L+3\rangle(0.2567)$ \\
\hline & & & & & $|H \rightarrow L ; H \rightarrow L+11\rangle(0.1361)$ \\
\hline & & & & & $|H \rightarrow L ; H \rightarrow L+8\rangle(0.1292)$ \\
\hline \multirow[t]{8}{*}{ II } & 2.48 & ${ }^{1} \Pi_{u}$ & 0.6411 & $\perp$ & $\left.H \rightarrow(L+2)_{1}\right\rangle(0.5041)$ \\
\hline & & & & & $\left.H \rightarrow(L+7)_{1}\right\rangle(0.4251)$ \\
\hline & & & & & $\left|H \rightarrow(L+2)_{1} ; H \rightarrow L+11\right\rangle(0.0848)$ \\
\hline & & & & & $\left|H \rightarrow(L+7)_{2} ; H \rightarrow L+11\right\rangle(0.0601)$ \\
\hline & 2.48 & ${ }^{1} \Pi_{u}$ & 0.6411 & $\perp$ & $\left|H \rightarrow(L+2)_{2}\right\rangle(0.5041)$ \\
\hline & & & & & $\left|H \rightarrow(L+7)_{1}\right\rangle(0.4251)$ \\
\hline & & & & & $\left|H \rightarrow(L+2)_{2} ; H \rightarrow L+11\right\rangle(0.0848)$ \\
\hline & & & & & $\left|H \rightarrow(L+7)_{1} ; H \rightarrow L+11\right\rangle(0.0601)$ \\
\hline \multirow[t]{8}{*}{ III } & 3.66 & ${ }^{1} \Pi_{u}$ & 0.1221 & $\perp$ & $\left|H \rightarrow(L+7)_{1}\right\rangle(0.4094)$ \\
\hline & & & & & $\left|H \rightarrow(L+2)_{2}\right\rangle(0.3656)$ \\
\hline & & & & & $\left|H \rightarrow(L+7)_{2}\right\rangle(0.2365)$ \\
\hline & & & & & $\left|H \rightarrow(L+2)_{1}\right\rangle(0.2130)$ \\
\hline & 3.66 & ${ }^{1} \Pi_{u}$ & 0.1221 & $\perp$ & $\left|H \rightarrow(L+7)_{2}\right\rangle(0.4094)$ \\
\hline & & & & & $\left|H \rightarrow(L+2)_{1}\right\rangle(0.3656)$ \\
\hline & & & & & $\left|H \rightarrow(L+7)_{1}\right\rangle(0.2365)$ \\
\hline & & & & & $\left|H \rightarrow(L+2)_{2}\right\rangle(0.2130)$ \\
\hline \multirow[t]{8}{*}{ IV } & 4.62 & ${ }^{1} \Pi_{u}$ & 0.0268 & $\perp$ & $\left|H \rightarrow(L+13)_{2}\right\rangle(0.3805)$ \\
\hline & & & & & $\left|H \rightarrow(L+7)_{1} ; H \rightarrow L+8\right\rangle(0.2572)$ \\
\hline & & & & & $\left|H \rightarrow(L+16)_{2}\right\rangle(0.2366)$ \\
\hline & & & & & $\left|(L+7)_{1} ; H \rightarrow L+11\right\rangle(0.2156)$ \\
\hline & 4.62 & ${ }^{1} \Pi_{u}$ & 0.0268 & $\perp$ & $\left|H \rightarrow(L+13)_{1}\right\rangle(0.3805)$ \\
\hline & & & & & $\left|H \rightarrow(L+7)_{2} ; H \rightarrow L+8\right\rangle(0.2572)$ \\
\hline & & & & & $\left|H \rightarrow(L+16)_{1}\right\rangle(0.2366)$ \\
\hline & & & & & $\left|(L+7)_{2} ; H \rightarrow L+11\right\rangle(0.2156)$ \\
\hline \multirow[t]{8}{*}{$\mathrm{V}$} & 5.46 & ${ }^{1} \Pi_{u}$ & 0.0115 & $\perp$ & $\left|H \rightarrow(L+13)_{2}\right\rangle(0.3608)$ \\
\hline & & & & & $\left|H \rightarrow L ; H \rightarrow(L+9)_{1}\right\rangle(0.2150)$ \\
\hline & & & & & $\left|(L+7)_{1} ; H \rightarrow L+8\right\rangle(0.2109)$ \\
\hline & & & & & $\left|(L+2)_{2} ; H \rightarrow L+8\right\rangle(0.1816)$ \\
\hline & 5.46 & ${ }^{1} \Pi_{u}$ & 0.0115 & $\perp$ & $\left|H \rightarrow(L+13)_{1}\right\rangle(0.3608)$ \\
\hline & & & & & $\left|H \rightarrow L ; H \rightarrow(L+9)_{2}\right\rangle(0.2150)$ \\
\hline & & & & & $\left|(L+7)_{2} ; H \rightarrow L+8\right\rangle(0.2109)$ \\
\hline & & & & & $\left.(L+2)_{1} ; H \rightarrow L+8\right\rangle(0.1816)$ \\
\hline
\end{tabular}


Table 2: Excitation energies $(E)$, many-particle wave functions of excited states corresponding to the peaks in the linear absorption spectrum of $\mathrm{Na}_{3}{ }^{+}$( $c f$. Fig. 8, in main text), along with the oscillator strengths. "in-plane" polarization implies photons polarized in the plane of the triangle, while "perpendicular" polarization implies photons polarized perpendicular to the plane of the triangle. For the doubly degenerate ${ }^{1} E^{\prime}$ symmetry, wave functions of both the states are presented. Rest of the information is same as in the caption of Table 1.

\begin{tabular}{|c|c|c|c|c|c|}
\hline Peak & $E(\mathrm{eV})$ & Symmetry & $f$ & Polarization & Wave Function \\
\hline \multirow[t]{8}{*}{ I } & 2.62 & ${ }^{1} E^{\prime}$ & 0.6254 & in-plane & $\left|H \rightarrow L_{1}\right\rangle(0.5199)$ \\
\hline & & & & & $\left.H \rightarrow L_{2}\right\rangle(0.3951)$ \\
\hline & & & & & $\left.H \rightarrow L_{1} ; H \rightarrow L+2\right\rangle(0.1031)$ \\
\hline & & & & & $\left.H \rightarrow L_{2} ; H \rightarrow L+2\right\rangle(0.0784)$ \\
\hline & 2.62 & ${ }^{1} E^{\prime}$ & 0.6254 & in-plane & $\left|H \rightarrow L_{2}\right\rangle(0.5199)$ \\
\hline & & & & & $\left|H \rightarrow L_{1}\right\rangle(0.3951)$ \\
\hline & & & & & $\left.H \rightarrow L_{2} ; H \rightarrow L+2\right\rangle(0.1031)$ \\
\hline & & & & & $\left.H \rightarrow L_{1} ; H \rightarrow L+2\right\rangle(0.0784)$ \\
\hline \multirow[t]{4}{*}{ II } & 3.25 & ${ }^{1} A_{2}^{\prime \prime}$ & 0.6344 & perpendicular & $|H \rightarrow L+1\rangle(0.6537)$ \\
\hline & & & & & $|H \rightarrow L+7\rangle(0.1246)$ \\
\hline & & & & & $|H \rightarrow L+1 ; H \rightarrow L+2\rangle(0.0792)$ \\
\hline & & & & & $|H \rightarrow L+1 ; H \rightarrow L+17\rangle(0.0778)$ \\
\hline \multirow[t]{8}{*}{ III } & 5.45 & ${ }^{1} E^{\prime}$ & 0.0239 & in-plane & $\left|H \rightarrow(L+5)_{2}\right\rangle(0.3364)$ \\
\hline & & & & & $\left.H \rightarrow(L+5)_{1}\right\rangle(0.2852)$ \\
\hline & & & & & $\left|H \rightarrow(L+13)_{1}\right\rangle(0.2626)$ \\
\hline & & & & & $\left|H \rightarrow L_{1} ; H \rightarrow L_{2}\right\rangle(0.2112)$ \\
\hline & 5.45 & ${ }^{1} E^{\prime}$ & 0.0239 & in-plane & $\left.H \rightarrow(L+5)_{1}\right\rangle(0.3364)$ \\
\hline & & & & & $\left.H \rightarrow(L+5)_{2}\right\rangle(0.2852)$ \\
\hline & & & & & $\left|H \rightarrow(L+13)_{2}\right\rangle(0.2626)$ \\
\hline & & & & & $\left|H \rightarrow L_{2} ; H \rightarrow L_{2}\right\rangle(0.2112)$ \\
\hline \multirow[t]{4}{*}{ IV } & 5.59 & ${ }^{1} A_{2}^{\prime \prime}$ & 0.0349 & perpendicular & $|H \rightarrow L+7\rangle(0.4707)$ \\
\hline & & & & & $|H \rightarrow L+16\rangle(0.3968)$ \\
\hline & & & & & $|H \rightarrow L+1 ; H \rightarrow L+2\rangle(0.2122)$ \\
\hline & & & & & $|H \rightarrow L+1\rangle(0.1175)$ \\
\hline
\end{tabular}


Table 3: Excitation energies, E, and many-particle wave functions of excited states corresponding to the peaks in the linear absorption spectrum of $\mathrm{Na}_{3}$ - isosceles triangular cluster ( $c f$. Fig. 9, in main text), along with the oscillator strengths $(f)$ of the transitions. Possible polarizations are: (a) in plane of the triangle along the perpendicular to the long arm (base) of the triangle (labeled: "in-plane, short axis"), (b) in plane of the triangle along the long arm (base) of the triangle (labeled "in-plane, long axis"), and (c) perpendicular to the plane of the triangle (labeled "perpendicular"). Rest of the notations are same as those in the previous tables.

\begin{tabular}{|c|c|c|c|c|c|}
\hline Peak & $\mathrm{E}(e V)$ & Symmetry & $f$ & Polarization & Wave Function \\
\hline \multirow[t]{4}{*}{$\overline{\mathrm{I}}$} & 0.4997 & ${ }^{2} A_{1}$ & 0.0065 & perpendicular & $H \rightarrow L\rangle(0.7815)$ \\
\hline & & & & & $|H \rightarrow L+5\rangle(0.3322)$ \\
\hline & & & & & $|H \rightarrow L+4\rangle(0.2014)$ \\
\hline & & & & & $H \rightarrow L+20\rangle(0.1985)$ \\
\hline \multirow[t]{4}{*}{ II } & 1.1108 & ${ }^{2} A_{1}$ & 0.0472 & perpendicular & $|H-1 \rightarrow H\rangle(0.6154)$ \\
\hline & & & & & $H \rightarrow L+14\rangle(0.4608)$ \\
\hline & & & & & $|H \rightarrow L+1\rangle(0.2831)$ \\
\hline & & & & & $|H \rightarrow L+4\rangle(0.2079)$ \\
\hline \multirow[t]{4}{*}{ III } & 1.6101 & ${ }^{2} A_{1}$ & 0.3336 & perpendicular & $|H \rightarrow L+1\rangle(0.5273)$ \\
\hline & & & & & $|H-1 \rightarrow H\rangle(0.4143)$ \\
\hline & & & & & $H \rightarrow L+10\rangle(0.3208)$ \\
\hline & & & & & $H \rightarrow L+16\rangle(0.3053)$ \\
\hline \multirow[t]{4}{*}{ IV } & 1.9944 & ${ }^{2} A_{1}$ & 0.4487 & perpendicular & $H \rightarrow L+16\rangle(0.4769)$ \\
\hline & & & & & $|H-1 \rightarrow H\rangle(0.4698)$ \\
\hline & & & & & $|H \rightarrow L+4\rangle(0.3876)$ \\
\hline & & & & & $|H \rightarrow L+5\rangle(0.2437)$ \\
\hline \multirow[t]{4}{*}{$\mathrm{V}$} & 2.2019 & ${ }^{2} B_{2}$ & 0.3970 & in-plane, long axis & $|H-1 \rightarrow L\rangle(0.4238)$ \\
\hline & & & & & $|H \rightarrow L+3\rangle(0.3707)$ \\
\hline & & & & & $H \rightarrow L+15\rangle(0.3521)$ \\
\hline & & & & & $H-1 \rightarrow H ; H \rightarrow L\rangle(0.2580)$ \\
\hline \multirow[t]{4}{*}{ VI } & 2.3539 & ${ }^{2} B_{2}$ & 0.2252 & in-plane, long axis & $|H \rightarrow L+6\rangle(0.4592)$ \\
\hline & & & & & $H \rightarrow L+19\rangle(0.4428)$ \\
\hline & & & & & $|H \rightarrow L+3\rangle(0.4043)$ \\
\hline & & & & & $|H-1 \rightarrow L\rangle(0.2602)$ \\
\hline \multirow[t]{4}{*}{ VII } & 2.5314 & ${ }^{2} A_{2}$ & 0.4157 & in-plane, short axis & $|H \rightarrow L+7\rangle(0.5306)$ \\
\hline & & & & & $H-1 \rightarrow L+2\rangle(0.3960)$ \\
\hline & & & & & $|H-1 \rightarrow L+11\rangle(0.3172)$ \\
\hline & & & & & $|H \rightarrow L+17\rangle(0.3119)$ \\
\hline \multirow[t]{4}{*}{ VIII } & 2.9243 & ${ }^{2} A_{2}$ & 0.2998 & in-plane, short axis & $|H \rightarrow L+2 ; H-1 \rightarrow H\rangle(0.4590)$ \\
\hline & & & & & $|H-1 \rightarrow L+11\rangle(0.3984)$ \\
\hline & & & & & $|H-1 \rightarrow L+2\rangle(0.3328)$ \\
\hline & & & & & $|H-1 \rightarrow H ; H \rightarrow L+11\rangle(0.2538)$ \\
\hline
\end{tabular}


Table 4: Excitation energies, E, and many-particle wave functions of excited states corresponding to the peaks in the linear absorption spectrum of $\mathrm{Na}_{3}$ equilateral triangle-shaped cluster ( $c f$. Fig. 10, in main text), along with the oscillator strengths $f$ of the transitions. Possible polarizations are: (a) in plane of the triangle (labeled "in-plane"), and (b) perpendicular to the plane of the triangle (labeled "perpendicular"). Rest of the notations are same as those in the previous tables.

\begin{tabular}{|c|c|c|c|c|}
\hline Peak & $E(\mathrm{eV})$ & $f$ & Polarization & Wave Function \\
\hline \multirow[t]{8}{*}{ I } & 1.1925 & 0.1755 & in-plane & $\overline{|H \rightarrow L+1\rangle(0.5108)}$ \\
\hline & & & & $H \rightarrow L+14\rangle(0.3805)$ \\
\hline & & & & $H \rightarrow L+16\rangle(0.3394)$ \\
\hline & & & & $|H-1 \rightarrow H\rangle(0.3102)$ \\
\hline & 1.2130 & 0.0250 & in-plane & $|H-1 \rightarrow L\rangle(0.5475)$ \\
\hline & & & & $H \rightarrow L+15\rangle(0.5307)$ \\
\hline & & & & $|H \rightarrow L+1\rangle(0.3235)$ \\
\hline & & & & $H \rightarrow L+16\rangle(0.2604)$ \\
\hline \multirow[t]{8}{*}{ II } & 2.0724 & 0.3470 & in-plane & $|H-1 \rightarrow L\rangle(0.5838)$ \\
\hline & & & & $H \rightarrow L+15\rangle(0.3604)$ \\
\hline & & & & $|H \rightarrow L+1\rangle(0.3591)$ \\
\hline & & & & $|H-1 \rightarrow L+3\rangle(0.2239)$ \\
\hline & 2.0743 & 0.3254 & in-plane & $|H \rightarrow L+7\rangle(0.4402)$ \\
\hline & & & & $|H-1 \rightarrow H\rangle(0.3985)$ \\
\hline & & & & $|H \rightarrow L ; H-1 \rightarrow L\rangle(0.3696)$ \\
\hline & & & & $|H \rightarrow L+16\rangle(0.3401)$ \\
\hline \multirow[t]{4}{*}{ III } & 2.3622 & 0.4090 & in-plane & $|H-1 \rightarrow L\rangle(0.7926)$ \\
\hline & & & & $|H-1 \rightarrow L+3\rangle(0.2389)$ \\
\hline & & & & $|H-1 \rightarrow L+19\rangle(0.1587)$ \\
\hline & & & & $|H-1 \rightarrow L ; H-1 \rightarrow L+22\rangle(0.1571)$ \\
\hline \multirow{4}{*}{ IV } & 2.9634 & 0.4938 & perpendicular & $|H-1 \rightarrow L+2\rangle(0.5975)$ \\
\hline & & & & $|H-1 \rightarrow L+11\rangle(0.4493)$ \\
\hline & & & & $|H-1 \rightarrow H\rangle(0.2695)$ \\
\hline & & & & $|H \rightarrow L+18\rangle(0.2600)$ \\
\hline \multirow[t]{4}{*}{$\mathrm{V}$} & 3.7223 & 0.0307 & in-plane & $|H \rightarrow L+3\rangle(0.4445)$ \\
\hline & & & & $|H \rightarrow L+4 ; H-1 \rightarrow L\rangle(0.3352)$ \\
\hline & & & & $|H-1 \rightarrow L+19\rangle(0.2945)$ \\
\hline & & & & $|H-1 \rightarrow L ; H \rightarrow L+20\rangle(0.2847)$ \\
\hline \multirow[t]{4}{*}{ VI } & 3.9622 & 0.0634 & perpendicular & $|H \rightarrow L+7\rangle(0.5908)$ \\
\hline & & & & $|H \rightarrow L+1 ; H-1 \rightarrow L+11\rangle(0.2889)$ \\
\hline & & & & $|H \rightarrow L+1 ; H-1 \rightarrow L+2\rangle(0.2815)$ \\
\hline & & & & $H-1 \rightarrow L+11\rangle(0.2295)$ \\
\hline
\end{tabular}


Table 5: Excitation energies, E, and many-particle wave functions of excited states corresponding to the peaks in the photoabsorption spectrum of the linear isomer of $\mathrm{Na}_{3}$ ( $c f$. Fig. 11, in main text), along with the oscillator strength $(f)$ of the transitions. Polarization corresponding to the transition is denoted as $\|$, if it is along the length of the molecule, and $\perp$, if it is perpendicular to it. Rest of the notations are same as those in the previous tables.

\begin{tabular}{|c|c|c|c|c|c|}
\hline Peak & $E(\mathrm{eV})$ & Symmetry & $f$ & Polarization & Wave Function \\
\hline \multirow[t]{4}{*}{$\mathrm{I}$} & 0.6414 & ${ }^{2} \Sigma_{g}$ & 0.0287 & 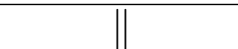 & $|H-1 \rightarrow H\rangle(0.8136)$ \\
\hline & & & & & $|H \rightarrow L\rangle(0.2675)$ \\
\hline & & & & & $|H \rightarrow L+4\rangle(0.2305)$ \\
\hline & & & & & $H \rightarrow L+5\rangle(0.1569)$ \\
\hline \multirow[t]{4}{*}{ II } & 1.3414 & ${ }^{2} \Sigma_{g}$ & 0.8917 & $\|$ & $|H \rightarrow L\rangle(0.6016)$ \\
\hline & & & & & $|H \rightarrow L+4\rangle(0.4124)$ \\
\hline & & & & & $|H-1 \rightarrow H\rangle(0.3893)$ \\
\hline & & & & & $H-1 \rightarrow H ; H-1 \rightarrow L\rangle(0.1857)$ \\
\hline \multirow[t]{8}{*}{ III } & 2.4268 & ${ }^{2} \Pi_{g}$ & 0.6994 & $\perp$ & $\left|H-1 \rightarrow(L+2)_{1}\right\rangle(0.4762)$ \\
\hline & & & & & $\left.H \rightarrow(L+3)_{1}\right\rangle(0.3944)$ \\
\hline & & & & & $\left|H \rightarrow(L+11)_{1}\right\rangle(0.3595)$ \\
\hline & & & & & $\left|H \rightarrow(L+2)_{1} ; H-1 \rightarrow H\right\rangle(0.3094)$ \\
\hline & 2.4268 & ${ }^{2} \Pi_{g}$ & 0.6994 & $\perp$ & $\left.H-1 \rightarrow(L+2)_{2}\right\rangle(0.4762)$ \\
\hline & & & & & $\left|H \rightarrow(L+3)_{2}\right\rangle(0.3944)$ \\
\hline & & & & & $\left|H \rightarrow(L+11)_{2}\right\rangle(0.3595)$ \\
\hline & & & & & $\left|H \rightarrow(L+2)_{2} ; H-1 \rightarrow H\right\rangle(0.3094)$ \\
\hline
\end{tabular}


Table 6: Excitation energies $(E)$, and many-particle wave functions of excited states corresponding to the peaks in the linear absorption spectrum of rhombus-shape $\mathrm{Na}_{4}$ cluster $(c f$. Fig. 12, in main text), along with the oscillator strengths $f$ of the transitions. The cluster is assumed to lie in the $x y$ plane, with $x$ and $y$ axes along the short, and long the diagonals, respectively, while the $z$ axis is assumed perpendicular to the plane of the molecule. Rest of the notations are same as in the previous tables.

\begin{tabular}{|c|c|c|c|c|c|}
\hline Peak & State & $E(\mathrm{eV})$ & $f$ & Polarization & Wave Function \\
\hline \multirow[t]{5}{*}{ I } & 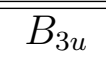 & 1.712 & 1.1477 & 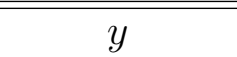 & $\overline{|H \rightarrow L+2\rangle(0.7232)}$ \\
\hline & & & & & $|H \rightarrow L+10\rangle(0.3832)$ \\
\hline & & & & & $|H \rightarrow L+20\rangle(0.1768)$ \\
\hline & & & & & $|H \rightarrow L+8\rangle(0.1720)$ \\
\hline & & & & & $|H \rightarrow L+2 ; H \rightarrow L+24\rangle(0.1330)$ \\
\hline \multirow[t]{3}{*}{ II } & $B_{1 u}$ & 2.090 & 0.0857 & $z$ & $|H \rightarrow L+22\rangle(0.4537)$ \\
\hline & & & & & $|H \rightarrow L+9\rangle(0.3546)$ \\
\hline & & & & & $|H \rightarrow L+8 ; H \rightarrow L+1\rangle(0.2771)$ \\
\hline \multirow[t]{5}{*}{ III } & $B_{2 u}$ & 2.470 & 0.7719 & $x$ & $|H \rightarrow L+6\rangle(0.5839)$ \\
\hline & & & & & $|H \rightarrow L+18\rangle(0.3464)$ \\
\hline & & & & & $|H \rightarrow L\rangle(0.3052)$ \\
\hline & & & & & $|H \rightarrow L+16\rangle(0.3018)$ \\
\hline & & & & & $H \rightarrow L+2 ; H \rightarrow L+3\rangle(0.1852)$ \\
\hline \multirow[t]{5}{*}{ IV } & $B_{1 u}$ & 2.699 & 0.6171 & $z$ & $|H \rightarrow L+9\rangle(0.5504)$ \\
\hline & & & & & $|H-1 \rightarrow L+1\rangle(0.3739)$ \\
\hline & & & & & $|H \rightarrow L+22\rangle(0.3032)$ \\
\hline & & & & & $|H-1 \rightarrow L+31\rangle(0.2119)$ \\
\hline & & & & & $|H \rightarrow L+2 ; H \rightarrow L+1\rangle(0.1626)$ \\
\hline \multirow[t]{3}{*}{$\mathrm{V}$} & $B_{1 u}$ & 3.010 & 0.1093 & $z$ & $|H-1 \rightarrow L+1\rangle(0.4178)$ \\
\hline & & & & & $|H \rightarrow L+8 ; H \rightarrow L+1\rangle(0.2934)$ \\
\hline & & & & & $|H \rightarrow L+8 ; H \rightarrow L+12\rangle(0.2740)$ \\
\hline \multirow[t]{3}{*}{ VI } & $B_{2 u}$ & 3.220 & 0.1641 & $x$ & $|H \rightarrow L+6\rangle(0.5619)$ \\
\hline & & & & & $|H \rightarrow L+18\rangle(0.4104)$ \\
\hline & & & & & $|H \rightarrow L+16\rangle(0.3258)$ \\
\hline \multirow[t]{5}{*}{ VI } & $B_{1 u}$ & 3.280 & 0.1371 & $z$ & $|H \rightarrow L+9\rangle(0.3237)$ \\
\hline & & & & & $|H-1 \rightarrow L+1\rangle(0.3092)$ \\
\hline & & & & & $|H \rightarrow L+19\rangle(0.2290)$ \\
\hline & & & & & $|H \rightarrow L+5 ; H \rightarrow L+12\rangle(0.2049)$ \\
\hline & & & & & $|H \rightarrow L+5 ; H \rightarrow L+1\rangle(0.1911)$ \\
\hline \multirow[t]{5}{*}{ VII } & $B_{1 u}$ & 3.900 & 0.0641 & $z$ & $|H-1 \rightarrow L+23\rangle(0.3563)$ \\
\hline & & & & & $|H-1 \rightarrow L+5\rangle(0.2860)$ \\
\hline & & & & & $|H \rightarrow L+24 ; H \rightarrow L+22\rangle(0.2348)$ \\
\hline & & & & & $|H \rightarrow L+8 ; H \rightarrow L+23\rangle(0.1733)$ \\
\hline & & & & & $|H \rightarrow L+7\rangle(0.1712)$ \\
\hline \multirow[t]{3}{*}{ VIII } & $B_{1 u}$ & 4.010 & 0.0532 & $z$ & $|H-1 \rightarrow L+12\rangle(0.4309)$ \\
\hline & & & & & $|H-1 \rightarrow L+1\rangle(0.3721)$ \\
\hline & & & & & $|H-1 \rightarrow L+5\rangle(0.2459)$ \\
\hline \multirow[t]{3}{*}{ IX } & $B_{1 u}$ & 4.670 & 0.0332 & $z$ & $|H \rightarrow L+8 ; H \rightarrow L+1\rangle(0.3437)$ \\
\hline & & & & & $|H-1 \rightarrow L+12\rangle(0.3051)$ \\
\hline & & & & & $|H \rightarrow L+35\rangle(0.2758)$ \\
\hline $\mathrm{X}$ & $B_{1}$ & 4.912 & 0.0452 & $z$ & $|H \rightarrow L+35\rangle(0.3861)$ \\
\hline
\end{tabular}


Table 7: Excitation energies, $E$, and many-particle wave functions of excited states corresponding to the peaks in the linear absorption spectrum of $\mathrm{Na}_{4}$, square-shaped cluster ( $c f$. Fig. 13, in main text), along with the oscillator strengths of the transitions. Rest of the information is same as in previous tables.

\begin{tabular}{|c|c|c|c|c|c|}
\hline Peak & $E(\mathrm{eV})$ & Symmetry & $f$ & Polarization & Wave Function \\
\hline \multirow[t]{8}{*}{ I } & 1.164 & ${ }^{1} E_{u}$ & 0.0107 & in-plane & $|H \rightarrow L ; H \rightarrow L+18\rangle(0.4144)$ \\
\hline & & & & & $|H \rightarrow L ; H \rightarrow L+4\rangle(0.3638)$ \\
\hline & & & & & $|H-1 \rightarrow L\rangle(0.3327)$ \\
\hline & & & & & $|H \rightarrow L+16\rangle(0.2510)$ \\
\hline & 1.166 & ${ }^{1} E_{u}$ & 0.0110 & in-plane & $|H \rightarrow L+1\rangle(0.4755)$ \\
\hline & & & & & $|H \rightarrow L+9\rangle(0.3948)$ \\
\hline & & & & & $|H-1 \rightarrow L ; H \rightarrow L\rangle(0.3165)$ \\
\hline & & & & & $|H \rightarrow L+18\rangle(0.2633)$ \\
\hline \multirow[t]{8}{*}{ II } & 2.005 & ${ }^{1} E_{u}$ & 1.0985 & in-plane & $|H \rightarrow L+4\rangle(0.4848)$ \\
\hline & & & & & $|H \rightarrow L+13\rangle(0.3791)$ \\
\hline & & & & & $|H \rightarrow L+4\rangle(0.3777)$ \\
\hline & & & & & $|H \rightarrow L+18\rangle(0.2474)$ \\
\hline & 2.015 & ${ }^{1} E_{u}$ & 1.0709 & in-plane & $|H \rightarrow L ; H \rightarrow L+1\rangle(0.5937)$ \\
\hline & & & & & $|H-1 \rightarrow L\rangle(0.2880)$ \\
\hline & & & & & $|H \rightarrow L ; H \rightarrow L+9\rangle(0.2826)$ \\
\hline & & & & & $|H \rightarrow L+1 ; H \rightarrow L+22\rangle(0.2122)$ \\
\hline \multirow[t]{8}{*}{ III } & 2.175 & ${ }^{1} E_{u}$ & 0.6775 & in-plane & $|H \rightarrow L+5\rangle(0.4769)$ \\
\hline & & & & & $|H \rightarrow L ; H \rightarrow L+4\rangle(0.4122)$ \\
\hline & & & & & $|H \rightarrow L+16\rangle(0.3209)$ \\
\hline & & & & & $|H \rightarrow L ; H \rightarrow L+13\rangle(0.2071)$ \\
\hline & 2.177 & ${ }^{1} E_{u}$ & 0.6833 & in-plane & $\mid H \rightarrow L ; H \rightarrow(L+5\rangle(0.4283)$ \\
\hline & & & & & $|H \rightarrow L+1\rangle(0.3461)$ \\
\hline & & & & & $|H \rightarrow L+4\rangle(0.3302)$ \\
\hline & & & & & $|H \rightarrow L+9\rangle(0.2805)$ \\
\hline \multirow[t]{8}{*}{ IV } & 2.482 & ${ }^{1} E_{u}$ & 0.4637 & in-plane & $|H-1 \rightarrow L\rangle(0.6015)$ \\
\hline & & & & & $|H \rightarrow L+5\rangle(0.3705)$ \\
\hline & & & & & $|H \rightarrow L ; H \rightarrow L+1\rangle(0.1639)$ \\
\hline & & & & & $|H \rightarrow L+4\rangle(0.1523)$ \\
\hline & 2.485 & ${ }^{1} E_{u}$ & 0.4375 & in-plane & $|H-1 \rightarrow L ; H \rightarrow L\rangle(0.4970)$ \\
\hline & & & & & $|H \rightarrow L ; H \rightarrow L+5\rangle(0.3376)$ \\
\hline & & & & & $|H \rightarrow L ; H-1 \rightarrow L+22\rangle(0.2057)$ \\
\hline & & & & & $|H \rightarrow L+5\rangle(0.1761)$ \\
\hline \multirow[t]{4}{*}{$\mathrm{V}$} & 2.752 & $A_{2 u}$ & 0.6634 & perpendicular & $|H \rightarrow L+8\rangle(0.4383)$ \\
\hline & & & & & $|H \rightarrow L ; H \rightarrow L+7\rangle(0.3968)$ \\
\hline & & & & & $|H-1 \rightarrow L+2\rangle(0.2655)$ \\
\hline & & & & & $\rightarrow L+23\rangle(0.2365)$ \\
\hline
\end{tabular}


Table 8: Excitation energies, $E$, and many-particle wave functions of excited states corresponding to the peaks in the linear absorption spectrum of $\mathrm{Na}_{5}+D_{2 d}$ structure ( $c f$. Fig. 14(b), in main text), along with the oscillator strength $f$ of the transitions. In our calculations, the two triangles of the structure are assumed to lie in the $y z$ and $x z$ planes, while $z$-axis is along the common perpendicular bisectors of the two triangles. Thus, $x y$-plane is perpendicular to the planes of both the triangles. Rest of the information is same as in previous tables.

\begin{tabular}{|c|c|c|c|c|c|}
\hline Peak & $E(\mathrm{eV})$ & Symmetry & $f$ & Polarization & Wave Function \\
\hline \multirow[t]{8}{*}{$\mathrm{I}$} & 1.770952 & ${ }^{1} E$ & 0.0636 & $x y$ & $\left|H \rightarrow L_{1}\right\rangle(0.6165)$ \\
\hline & & & & & $\left|H \rightarrow L_{2}\right\rangle(0.5691)$ \\
\hline & & & & & $\left.H \rightarrow L_{1} ; H-1 \rightarrow L+1\right\rangle(0.1363)$ \\
\hline & & & & & $\left.H \rightarrow L_{2} ; H-1 \rightarrow L+1\right\rangle(0.1258)$ \\
\hline & 1.770952 & ${ }^{1} E$ & 0.0636 & $x y$ & $\left|H \rightarrow L_{2}\right\rangle(0.6165)$ \\
\hline & & & & & $\left.H \rightarrow L_{1}\right\rangle(0.5691)$ \\
\hline & & & & & $\left.H \rightarrow L_{2} ; H-1 \rightarrow L+1\right\rangle(0.1363)$ \\
\hline & & & & & $\left.H \rightarrow L_{1} ; H-1 \rightarrow L+1\right\rangle(0.1258)$ \\
\hline \multirow[t]{4}{*}{ II } & 2.243351 & ${ }^{1} B_{2}$ & 1.3390 & $z$ & $|H \rightarrow(L+1)\rangle(0.8954)$ \\
\hline & & & & & $|H-1 \rightarrow(L+3)\rangle(0.1582)$ \\
\hline & & & & & $|H \rightarrow(L+1) ; H \rightarrow(L+3)\rangle(0.1158)$ \\
\hline & & & & & $|H \rightarrow(L+8)\rangle(0.0905)$ \\
\hline \multirow[t]{8}{*}{ III } & 2.6766435 & ${ }^{1} E$ & 0.6144 & $x y$ & $\left|H \rightarrow(L+2)_{2}\right\rangle(0.4478)$ \\
\hline & & & & & $\left|H-1 \rightarrow L_{1}\right\rangle(0.4014)$ \\
\hline & & & & & $\left|H-1 \rightarrow L_{2}\right\rangle(0.3116)$ \\
\hline & & & & & $\left|H-1 \rightarrow L_{1}\right\rangle(0.2792)$ \\
\hline & 2.6766435 & ${ }^{1} E$ & 0.6144 & $x y$ & $\left|H \rightarrow(L+2)_{1}\right\rangle(0.4478)$ \\
\hline & & & & & $\left|H-1 \rightarrow L_{2}\right\rangle(0.4014)$ \\
\hline & & & & & $\left|H-1 \rightarrow L_{1}\right\rangle(0.3116)$ \\
\hline & & & & & $\left|H-1 \rightarrow L_{2}\right\rangle(0.2792)$ \\
\hline \multirow[t]{8}{*}{ IV } & 3.316498 & ${ }^{1} E$ & 0.5247 & $x y$ & $\left|H-1 \rightarrow(L+2)_{2}\right\rangle(0.3950)$ \\
\hline & & & & & $\left|H-1 \rightarrow(L+2)_{1}\right\rangle(0.3233)$ \\
\hline & & & & & $\left|H \rightarrow(L+2)_{2}\right\rangle(0.3050)$ \\
\hline & & & & & $\left|H \rightarrow(L+2)_{1}\right\rangle(0.2495)$ \\
\hline & 3.316498 & ${ }^{1} E$ & 0.5247 & $x y$ & $\left|H-1 \rightarrow(L+2)_{1}\right\rangle(0.3950)$ \\
\hline & & & & & $\left|H-1 \rightarrow(L+2)_{2}\right\rangle(0.3233)$ \\
\hline & & & & & $\left|H \rightarrow(L+2)_{1}\right\rangle(0.3050)$ \\
\hline & & & & & $\left|H \rightarrow(L+2)_{2}\right\rangle(0.2495)$ \\
\hline \multirow[t]{8}{*}{$\mathrm{V}$} & 3.966769 & ${ }^{1} E$ & 0.0250 & $x y$ & $\left|H-1 \rightarrow(L+2)_{1}\right\rangle(0.3039)$ \\
\hline & & & & & $\left|H-1 \rightarrow(L+2)_{2}\right\rangle(0.3025)$ \\
\hline & & & & & $\left.H \rightarrow L_{1} ; H \rightarrow L+3\right\rangle(0.2164)$ \\
\hline & & & & & $\left|H \rightarrow L_{2} ; H \rightarrow L+3\right\rangle(0.2154)$ \\
\hline & 3.966769 & ${ }^{1} E$ & 0.0250 & $x y$ & $\left|H-1 \rightarrow(L+2)_{2}\right\rangle(0.3039)$ \\
\hline & & & & & $\left|H-1 \rightarrow(L+2)_{1}\right\rangle(0.3025)$ \\
\hline & & & & & $\left.H \rightarrow L_{2} ; H \rightarrow L+3\right\rangle(0.2164)$ \\
\hline & & & & & $\left|H \rightarrow L_{1} ; H \rightarrow L+3\right\rangle(0.2154)$ \\
\hline
\end{tabular}


Table 9: Excitation energies, $E$, and many-particle wave functions of excited states corresponding to the peaks in the linear absorption spectrum of $\mathrm{Na}_{5}{ }^{+} \mathrm{D}_{2 h}$ structure ( $c f$. Fig. 14(a), in main text), along with the oscillator strength $f$ of the transitions. Rest of the information is same as in previous tables.

\begin{tabular}{|c|c|c|c|c|c|}
\hline Peak & $E(\mathrm{eV})$ & Symmetry & $f$ & Polarization & Wave Function \\
\hline \hline I & 2.149 & ${ }^{1} B_{2 u}$ & 1.3471 & in-plane & $|H \rightarrow L+1\rangle(0.8855)$ \\
\hline & & & & & $|H-1 \rightarrow H ; H \rightarrow L+5\rangle(0.1484)$ \\
\hline & & & & & $|H \rightarrow L+1 ; H \rightarrow L+5\rangle(0.1148)$ \\
\hline & & & & & $|H \rightarrow L+13\rangle(0.0863)$ \\
\hline II & 2.508 & ${ }^{1} B_{3 u}$ & 1.1284 & in-plane & $|H-1 \rightarrow H ; H \rightarrow L\rangle(0.6883)$ \\
\hline & & & & & $|H \rightarrow L+2\rangle(0.4827)$ \\
\hline & & & & & $|H \rightarrow L ; H \rightarrow L+1\rangle(0.1960)$ \\
\hline & & & & & $|H-1 \rightarrow L ; H-1 \rightarrow L+1\rangle(0.1770)$ \\
\hline III & 3.215 & ${ }^{1} B_{1 u}$ & 1.1321 & perpendicular & $|H-1 \rightarrow H ; H \rightarrow L+3\rangle(0.6405)$ \\
\hline & & & & & $|H \rightarrow L+6\rangle(0.5829)$ \\
\hline & & & & & $|H-1 \rightarrow L+1 ; H-1 \rightarrow L+3\rangle(0.1497)$ \\
\hline & & & & & $|H \rightarrow L+20\rangle(0.1264)$ \\
\hline IV & 3.626 & ${ }^{1} B_{3 u}$ & 0.0826 & in-plane & $|H-1 \rightarrow H ; H \rightarrow L+7\rangle(0.3928)$ \\
\hline & & & & & $|H \rightarrow L+31\rangle(0.3100)$ \\
\hline & & & & & $|H-1 \rightarrow L+1 ; H \rightarrow L+2\rangle(0.2732)$ \\
\hline & & & & & $|H \rightarrow L ; H \rightarrow L+1\rangle(0.2664)$ \\
\hline
\end{tabular}

Table 10: Excitation energies, $E$, and many-particle wave functions of excited states corresponding to the peaks in the linear absorption spectrum of $\mathrm{Na}_{5}{ }^{+} C_{2 v}$ structure ( $c f$. Fig. 14(a), in main text), along with the oscillator strength $f$ of the transitions. Rest of the information is same as in previous tables.

\begin{tabular}{|c|c|c|c|c|c|}
\hline Peak & $E(\mathrm{eV})$ & Symmetry & $f$ & Polarization & Wave Function \\
\hline \hline I & 2.096397 & ${ }^{1} B_{2}$ & 1.0294 & in-plane & $|H \rightarrow L+1\rangle(0.8189)$ \\
\hline & & & & & $|H \rightarrow L+1 ; H \rightarrow L+2\rangle(0.1855)$ \\
\hline & & & & & $|H \rightarrow L ; H \rightarrow L+2\rangle(0.1769)$ \\
\hline & & & & & $|H \rightarrow L ; H-1 \rightarrow L\rangle(0.1612)$ \\
\hline II & 2.400917 & ${ }^{1} A_{1}$ & 1.3692 & in-plane & $|H \rightarrow L+2\rangle(0.6908)$ \\
\hline & & & & & $|H-1 \rightarrow L\rangle(0.5297)$ \\
\hline & & & & & $|H-1 \rightarrow L ; H-1 \rightarrow L ; H \rightarrow L+2\rangle(0.1551)$ \\
\hline & & & & & $|H-1 \rightarrow L ; H \rightarrow L+2 ; H \rightarrow L+2\rangle(0.1309)$ \\
\hline III & 3.208789 & ${ }^{1} B_{1}$ & 1.2917 & perpendicular & $|H \rightarrow L+5\rangle(0.6425)$ \\
\hline & & & & & $|H-1 \rightarrow L+3\rangle(0.6185)$ \\
\hline & & & & & $|H \rightarrow L+20\rangle(0.1186)$ \\
\hline & & & & & $|H-1 \rightarrow L+7\rangle(0.1026)$ \\
\hline
\end{tabular}


Table 11: Excitation energies, $E$, and many-particle wave functions of excited states corresponding to the peaks in the linear absorption spectrum $\mathrm{Na}$ of $\mathrm{Na}_{5}{ }^{+} D_{3 h}$ structure $(c f$. Fig. 14(a), in main text), along with the oscillator strength $f$ of the transitions In the wave function, the bracketed numbers are the CI coefficients of a given electronic configuration. Given the trigonal bipyramidal structure of the cluster ( $c f$. Fig. $6(\mathrm{j})$ ), and ${ }^{1} A_{1}^{\prime}$ symmetry of the ground state, optically active excited states are of doubly degenerate ${ }^{1} E^{\prime}$ symmetry with polarization along the common base of the pyramids, and of ${ }^{1} A_{2}^{\prime \prime}$ symmetry, with polarization perpendicular to the base. We refer to their polarizations as "in-plane", and "perpendicular", respectively. Rest of the information is same as in previous tables.

\begin{tabular}{|c|c|c|c|c|c|}
\hline Peak & $E(\mathrm{eV})$ & Symmetry & $f$ & Polarization & Wave Function \\
\hline \hline I & 2.227 & ${ }^{1} A_{2}^{\prime \prime}$ & 0.9568 & perpendicular & $|H \rightarrow L+1\rangle(0.7991)$ \\
\hline & & & & & $|H \rightarrow L+6\rangle(0.3394)$ \\
\hline & & & & & $|H \rightarrow L+8\rangle(0.1986)$ \\
\hline & & & & & $\left|H \rightarrow L_{2} ; H \rightarrow(L+3)_{1}\right\rangle(0.1332)$ \\
\hline II & 3.163 & ${ }^{1} E^{\prime}$ & 1.0761 & in-plane & $\left|H \rightarrow(L+3)_{1}\right\rangle(0.7281)$ \\
\hline & & & & & $\left|H-1 \rightarrow H ; H \rightarrow L_{2}\right\rangle(0.3847)$ \\
\hline & & & & & $\left|H \rightarrow L_{2} ; H \rightarrow L+1\right\rangle(0.2311)$ \\
\hline & & & & & $\left|H \rightarrow L_{2} ; H \rightarrow L+2\right\rangle(0.2020)$ \\
\hline & 3.163 & ${ }^{1} E^{\prime}$ & 1.0761 & in-plane & $\left|H \rightarrow(L+3)_{2}\right\rangle(0.7281)$ \\
\hline & & & & & $\left|H-1 \rightarrow H ; H \rightarrow L_{1}\right\rangle(0.3847)$ \\
\hline & & & & & $\left|H \rightarrow L_{1} ; H \rightarrow L+1\right\rangle(0.2311)$ \\
\hline & & & & & $\left|H \rightarrow L_{1} ; H \rightarrow L+2\right\rangle(0.2019)$ \\
\hline
\end{tabular}


Table 12: Excitation energies, E, and many-particle wave functions of excited states corresponding to the peaks in the linear absorption spectrum of $\mathrm{Na}_{5^{-}}$planar $\left(C_{2 v}\right)$ structure $(c f$. Fig. 15(a), in main text), along with the oscillator strengths $f$ of the transitions. Given the ${ }^{2} A_{1}^{\prime}$ symmetry of the ground state, optically active excited states are of ${ }^{2} B_{1}$ and ${ }^{2} A_{1}$ type with polarizations in plane of the cluster, and ${ }^{2} B_{2}$ type, with polarization perpendicular to it. We refer to these polarizations as "in-plane", and "perpendicular", respectively. Rest of the information is same as in previous tables.

\begin{tabular}{|c|c|c|c|c|c|}
\hline Peak & $E(\mathrm{eV})$ & Symmetry & $f$ & Polarization & Wave Function \\
\hline \multirow[t]{4}{*}{ I } & 1.15 & ${ }^{2} B_{1}$ & 0.0210 & in-plane & $H-2 \rightarrow H)\rangle(0.4052)$ \\
\hline & & & & & $H \rightarrow L\rangle(0.2818)$ \\
\hline & & & & & $H-1 \rightarrow L+2\rangle(0.2694)$ \\
\hline & & & & & $H-1 \rightarrow L+10\rangle(0.2577)$ \\
\hline \multirow[t]{4}{*}{ II } & 1.96 & ${ }^{2} A_{1}$ & 0.5896 & in-plane & $|H-1 \rightarrow L\rangle(0.5565)$ \\
\hline & & & & & $H-1 \rightarrow L+21\rangle 0.2734)$ \\
\hline & & & & & $H-1 \rightarrow L+8\rangle(0.2634)$ \\
\hline & & & & & $H-1 \rightarrow L+4\rangle(0.1669)$ \\
\hline \multirow[t]{4}{*}{ III } & 2.31 & ${ }^{2} B_{1}$ & 0.7687 & in-plane & $|H \rightarrow L+4\rangle(0.3359)$ \\
\hline & & & & & $|H-1 \rightarrow L+2\rangle(0.3075)$ \\
\hline & & & & & $H-2 \rightarrow H)\rangle(0.2867)$ \\
\hline & & & & & $|H \rightarrow L+14\rangle(0.2278)$ \\
\hline \multirow[t]{4}{*}{ IV } & 2.76 & ${ }^{2} B_{2}$ & 0.2170 & perpendicular & $|H \rightarrow L+7\rangle(0.3108)$ \\
\hline & & & & & $|H-1 \rightarrow L+6\rangle(0.2691)$ \\
\hline & & & & & $|H-1 \rightarrow L+1 ; H \rightarrow L+2\rangle(0.2060)$ \\
\hline & & & & & $|H-1 \rightarrow L+1 ; H \rightarrow L+10\rangle(0.2016)$ \\
\hline
\end{tabular}


Table 13: Excitation energies, E, and many-particle wave functions of excited states corresponding to the peaks in the linear absorption spectrum of $\mathrm{Na}_{5}$ bipyramid $\left(C_{2 v}\right)$ structure (cf. Fig. 15(b), in main text), along with the oscillator strengths $f$ of the transitions. Given the trigonal bipyramidal structure of the cluster ( $c f$. Fig. $6(\mathrm{~m})$ ), and ${ }^{2} B_{1}$ symmetry of the ground state, optically active excited states are of ${ }^{2} A_{2}$ and ${ }^{2} B_{1}$ symmetry with polarization along the common base of the pyramids, and of ${ }^{2} A_{1}$ symmetry, with polarization perpendicular to the base. We refer to their polarizations as "in-plane", and "perpendicular", respectively. Rest of the information is same as in previous tables.

\begin{tabular}{|c|c|c|c|c|c|}
\hline Peak & $E(\mathrm{eV})$ & Symmetry & f & Polarization & Wave Function \\
\hline \multirow[t]{4}{*}{ 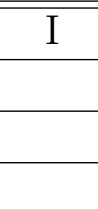 } & 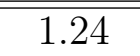 & $\overline{2}{ }^{2} B_{1}$ & 0.0515 & in-plane & $\mid \overline{|H-1 \rightarrow H ; H-1 \rightarrow L\rangle(0.4700)}$ \\
\hline & & & & & $|H \rightarrow L+16\rangle(0.3747)$ \\
\hline & & & & & $|H-1 \rightarrow H ; H-2 \rightarrow H-1\rangle(0.3036)$ \\
\hline & & & & & $|H \rightarrow L+2\rangle(0.2931)$ \\
\hline \multirow[t]{6}{*}{ II } & 1.57 & ${ }^{2} A_{1}$ & 0.2306 & perpendicular & $H-1 \rightarrow H ; H \rightarrow L+16\rangle(0.5187)$ \\
\hline & & & & & $|H-1 \rightarrow H ; H \rightarrow L+1\rangle(0.4365)$ \\
\hline & & & & & $|H-1 \rightarrow H ; H \rightarrow L+2\rangle(0.3958)$ \\
\hline & & & & & $|H \rightarrow L+6\rangle(0.4517)$ \\
\hline & & & & & $|H \rightarrow L+19\rangle(0.4123)$ \\
\hline & & & & & $|H-1 \rightarrow H ; H-2 \rightarrow L+1\rangle(0.4070)$ \\
\hline \multirow[t]{4}{*}{ III } & 2.02 & ${ }^{2} A_{1}$ & 0.6558 & perpendicular & $|H-1 \rightarrow H ; H \rightarrow L+2\rangle(0.4562)$ \\
\hline & & & & & $|H-1 \rightarrow H ; H \rightarrow L+1\rangle(0.4040)$ \\
\hline & & & & & $|H-1 \rightarrow L ; H \rightarrow L+16\rangle(0.2540)$ \\
\hline & & & & & $|H \rightarrow L+6\rangle(0.2391)$ \\
\hline \multirow[t]{4}{*}{ IV } & 2.14 & ${ }^{2} A_{1}$ & 0.6381 & perpendicular & $H-1 \rightarrow H ; H \rightarrow L+2\rangle(0.4266)$ \\
\hline & & & & & $H-1 \rightarrow H ; H \rightarrow L+8\rangle(0.3772)$ \\
\hline & & & & & $|H-1 \rightarrow H ; H \rightarrow L+1\rangle(0.3019)$ \\
\hline & & & & & $|H \rightarrow L+6\rangle(0.2752)$ \\
\hline \multirow[t]{4}{*}{$\mathrm{V}$} & 2.44 & ${ }^{2} B_{1}$ & 0.5725 & in-plane & $|H-1 \rightarrow L+6\rangle(0.4432)$ \\
\hline & & & & & $|H-1 \rightarrow H ; H-1 \rightarrow L+16\rangle(0.3314)$ \\
\hline & & & & & $|H-1 \rightarrow H ; H-1 \rightarrow L+2\rangle(0.3175)$ \\
\hline & & & & & $|H \rightarrow L+2\rangle(0.2603)$ \\
\hline
\end{tabular}


Table 14: Excitation energies, $E$, and many-particle wave functions of excited states corresponding to the peaks in the linear absorption spectrum of $\mathrm{Na}_{6} C_{5 v}$ structure ( $c f$. Fig. 16(a), in main text), along with the oscillator strengths $f$ of the transitions. Given the ${ }^{1} A_{1}$ symmetry of the ground state, optically active excited states are of doubly degenerate ${ }^{1} E_{1}$ symmetry with polarization along the basal plane of the pyramid, and of ${ }^{1} A_{1}$ symmetry, with the polarization perpendicular to it. We refer to these polarizations as "in-plane", and "perpendicular", respectively. Because of the $C_{5 v}$ symmetry, the cluster has doubly-degenerate HOMOs indicated as $H_{1}$ and $H_{2}$. Rest of the information is same as in previous tables.

\begin{tabular}{|c|c|c|c|c|c|}
\hline Peak & $E(\mathrm{eV})$ & Symmetry & $f$ & Polarization & Wave Function \\
\hline \hline I & 1.19 & ${ }^{1} E_{1}$ & 0.0097 & in-plane & $\left|H_{2} \rightarrow L\right\rangle(0.7419)$ \\
\hline & & & & & $\left|H_{2} \rightarrow L+5\right\rangle(0.3170)$ \\
\hline & & & & & $\left|H_{2} \rightarrow L+2\right\rangle(0.2526)$ \\
\hline & & & & & $\left|H_{2} \rightarrow L+12\right\rangle(0.1383)$ \\
\hline & 1.19 & ${ }^{1} E_{1}$ & 0.0097 & in-plane & $\left|H_{1} \rightarrow L\right\rangle(0.7420)$ \\
\hline & & & & & $\left|H_{1} \rightarrow L+5\right\rangle(0.3170)$ \\
\hline & & & & & $\left|H_{1} \rightarrow L+2\right\rangle(0.2526)$ \\
\hline & & & & & $\left|H_{1} \rightarrow L+12\right\rangle(0.1383)$ \\
\hline II & 2.150 & ${ }^{1} E_{1}$ & 1.4732 & in-plane & $\left|H_{1} \rightarrow(L+1)_{2}\right\rangle(0.4811)$ \\
\hline & & & & & $\left|H_{2} \rightarrow(L+1)_{1}\right\rangle(0.4808)$ \\
\hline & & & & & $\left|H_{1} \rightarrow L\right\rangle(0.3100)$ \\
\hline & & & & & $\left|H_{1} \rightarrow(L+2)\right\rangle(0.3028)$ \\
\hline & 2.150 & ${ }^{1} E_{1}$ & 1.4732 & in-plane & $\left|H_{1} \rightarrow(L+1)_{1}\right\rangle(0.4811)$ \\
\hline & & & & & $\left|H_{2} \rightarrow(L+1)_{2}\right\rangle(0.4808)$ \\
\hline & & & & & $\left|H_{2} \rightarrow L\right\rangle(0.3100)$ \\
\hline & & & & & $\left|H_{2} \rightarrow(L+2)\right\rangle(0.3028)$ \\
\hline III & 2.527 & ${ }^{1} E_{1}$ & 0.2422 & in-plane & $\left|H_{1} \rightarrow(L+4)_{3}\right\rangle(0.4774)$ \\
\hline & & & & & $\left|H_{1} \rightarrow L+17\right\rangle(0.4643)$ \\
\hline & & & & & $\left|H_{1} \rightarrow(L+4)_{1}\right\rangle(0.3869)$ \\
\hline & & & & & $\left|H_{1} \rightarrow(L+1)_{2}\right\rangle(0.1903)$ \\
\hline & 2.527 & ${ }^{1} E_{1}$ & 0.2422 & in-plane & $\left|H_{2} \rightarrow(L+4)_{3}\right\rangle(0.4774)$ \\
\hline & & & & & $\left|H_{2} \rightarrow L+17\right\rangle(0.4643)$ \\
\hline & & & & & $\left|H_{2} \rightarrow(L+4)_{1}\right\rangle(0.3869)$ \\
\hline & & & & & $\left|H_{1} \rightarrow(L+1)_{1}\right\rangle(0.1903)$ \\
\hline IV & 2.786 & ${ }^{1} A_{1}$ & 0.4687 & perpendicular & $\left|H_{1} \rightarrow(L+3)_{1}\right\rangle(0.4135)$ \\
\hline & & & & & $\left|H_{2} \rightarrow(L+3)_{3}\right\rangle(0.4133)$ \\
\hline & & & & & $\left|H_{1} \rightarrow(L+4)_{2}\right\rangle(0.3280)$ \\
\hline & & & & & $\left|H_{2} \rightarrow(L+4)_{1}\right\rangle(0.2549)$ \\
\hline & & & & &
\end{tabular}


Table 15: Excitation energies, $E$, and many-particle wave functions of excited states corresponding to the peaks in the linear absorption spectrum of $\mathrm{Na}_{6} D_{3 h^{-}}$planar structure ( $c f$. Fig. 16(a), in main text), along with the oscillator strength $f$ of the transitions. Given the ${ }^{1} A_{1}^{\prime}$ symmetry of the ground state, optically active excited states are of doubly degenerate ${ }^{1} E^{\prime}$ symmetry with polarization in plane of the cluster, and of ${ }^{1} A_{2}^{\prime \prime}$ symmetry, with polarization perpendicular to it. We refer to these polarizations as "in-plane", and "perpendicular", respectively. Because of the $D_{3 h}$ symmetry, the cluster has doubly-degenerate HOMOs indicated as $H_{1}$ and $H_{2}$. Rest of the information is same as in previous tables.

\begin{tabular}{|c|c|c|c|c|c|c|}
\hline Peak & State & $E(\mathrm{eV})$ & Symmetry & Osci. Strength $(f)$ & Polarization & Wave Function \\
\hline \multirow[t]{8}{*}{ I } & & 1.36 & ${ }^{1} E^{\prime}$ & 0.1326 & in-plane & $\left|H_{1} \rightarrow L\right\rangle(0.5247)$ \\
\hline & & & & & & $\left|H_{2} \rightarrow L\right\rangle(0.3580)$ \\
\hline & & & & & & $\left|H_{1} \rightarrow L+4\right\rangle(0.2071)$ \\
\hline & & & & & & $\left|H_{1} \rightarrow(L+1)_{1}\right\rangle(0.1855)$ \\
\hline & & 1.36 & ${ }^{1} E^{\prime}$ & 0.1326 & & $\left|H_{2} \rightarrow L\right\rangle(0.5247)$ \\
\hline & & & & & & $\left|H_{1} \rightarrow L\right\rangle(0.3580)$ \\
\hline & & & & & & $\left|H_{2} \rightarrow L+4\right\rangle(0.2071)$ \\
\hline & & & & & & $\left|H_{2} \rightarrow(L+1)_{1}\right\rangle(0.1855)$ \\
\hline \multirow[t]{8}{*}{ II } & & 1.94 & ${ }^{1} E^{\prime}$ & 2.6326 & in-plane & $\left|H_{1} \rightarrow L\right\rangle(0.3499)$ \\
\hline & & & & & & $\left|H_{2} \rightarrow L\right\rangle(0.3055)$ \\
\hline & & & & & & $\left|H_{2} \rightarrow(L+1)_{2}\right\rangle(0.2989)$ \\
\hline & & & & & & $\left|H_{1} \rightarrow(L+1)_{1}\right\rangle(0.2989)$ \\
\hline & & 1.94 & ${ }^{1} E^{\prime}$ & 2.6325 & & $\left|H_{2} \rightarrow L\right\rangle(0.3499)$ \\
\hline & & & & & & $\left|H_{1} \rightarrow L\right\rangle(0.3055)$ \\
\hline & & & & & & $\left|H_{2} \rightarrow(L+1)_{1}\right\rangle(0.2989)$ \\
\hline & & & & & & $\left|H_{1} \rightarrow(L+1)_{2}\right\rangle(0.2989)$ \\
\hline \multirow[t]{8}{*}{ III } & & 2.49 & ${ }^{1} E^{\prime}$ & 0.0748 & in-plane & $\left|H_{1} \rightarrow(L+3)_{2}\right\rangle(0.2471)$ \\
\hline & & & & & & $\left|H_{2} \rightarrow(L+3)_{1}\right\rangle(0.2471)$ \\
\hline & & & & & & $\left|H_{2} \rightarrow(L+3)_{2}\right\rangle(0.2028)$ \\
\hline & & & & & & $\left|H_{1} \rightarrow(L+3)_{1}\right\rangle(0.2028)$ \\
\hline & & 2.49 & ${ }^{1} E^{\prime}$ & 0.0748 & in-plane & $\left|H_{2} \rightarrow(L+3)_{2}\right\rangle(0.2471)$ \\
\hline & & & & & & $\left|H_{1} \rightarrow(L+3)_{1}\right\rangle(0.2471)$ \\
\hline & & & & & & $\left|H_{1} \rightarrow(L+3)_{2}\right\rangle(0.2028)$ \\
\hline & & & & & & $\left|H_{2} \rightarrow(L+3)_{1}\right\rangle(0.2028)$ \\
\hline \multirow[t]{4}{*}{ IV } & & 2.81 & ${ }^{1} A_{2}^{\prime \prime}$ & 0.8056 & perpendicular & $\left|H_{2} \rightarrow(L+5)_{2}\right\rangle(0.4686)$ \\
\hline & & & & & & $\left|H_{1} \rightarrow(L+5)_{1}\right\rangle(0.4685)$ \\
\hline & & & & & & $|H-1 \rightarrow L+2\rangle(0.3190)$ \\
\hline & & & & & & $\left|H_{2} \rightarrow(L+17)_{2}\right\rangle(0.1671)$ \\
\hline
\end{tabular}


Table 16: Excitation energies, $E$, and many-particle wave functions of excited states corresponding to the peaks in the linear absorption spectrum of $\mathrm{Na}_{6}, D_{4 h}$ structure ( $c f$. Fig. 16(b), in main text), along with the oscillator strength $f$ of the transitions. Because of the $D_{4 h}$ symmetry, the cluster has doubly-degenerate HOMOs indicated as $H_{1}$ and $H_{2}$. Given the bipyramidal structure of the cluster ( $c f$. Fig. 6(p) of the main text) and ${ }^{1} A_{1 g}$ symmetry of the ground state, optically active excited states are of doubly degenerate ${ }^{1} E_{u}$ symmetry with polarization along the common base of the pyramids, and of ${ }^{1} A_{2 u}$ symmetry, with polarization perpendicular to the base. We refer to these polarizations as "in-plane", and "perpendicular", respectively. Rest of the information is same as in previous tables.

\begin{tabular}{|c|c|c|c|c|c|}
\hline Peak & $E(\mathrm{eV})$ & Symmetry & $f$ & Polarization & Wave Function \\
\hline \hline $\mathrm{I}$ & 1.647 & ${ }^{1} E_{u}$ & 0.2786 & in-plane & $\left|H_{2} \rightarrow(L+1)\right\rangle(0.4269)$ \\
\hline & & & & & $\left|H_{2} \rightarrow(L+2)\right\rangle(0.3583)$ \\
\hline & & & & & $\left|H_{1} \rightarrow(L+6)\right\rangle(0.3087)$ \\
\hline & & & & & $\left|H_{2} \rightarrow(L+9)\right\rangle(0.2703)$ \\
\hline & 1.647 & ${ }^{1} E_{u}$ & 0.2786 & in-plane & $\left|H_{1} \rightarrow(L+1)\right\rangle(0.4269)$ \\
\hline & & & & & $\left|H_{1} \rightarrow(L+2)\right\rangle(0.3583)$ \\
\hline & & & & & $\left|H_{2} \rightarrow(L+6)\right\rangle(0.3087)$ \\
\hline & & & & & $\left|H_{1} \rightarrow(L+9)\right\rangle(0.2703)$ \\
\hline II & 1.90 & ${ }^{1} E_{u}$ & 0.0421 & in-plane & $\left|H_{2} \rightarrow(L+5)\right\rangle(0.4200)$ \\
\hline & & & & & $\left|H_{2} \rightarrow(L+15)\right\rangle(0.3930)$ \\
\hline & & & & & $\left|H_{1} \rightarrow(L+5)\right\rangle(0.2992)$ \\
\hline & & & & & $\left|H_{1} \rightarrow(L+15)\right\rangle(0.2799)$ \\
\hline & 1.90 & ${ }^{1} E_{u}$ & 0.0421 & in-plane & $\left|H_{1} \rightarrow(L+5)\right\rangle(0.4200)$ \\
\hline & & & & & $\left|H_{1} \rightarrow(L+15)\right\rangle(0.3930)$ \\
\hline & & & & & $\left|H_{2} \rightarrow(L+5)\right\rangle(0.2992)$ \\
\hline & & & & & $\left|H_{2} \rightarrow(L+15)\right\rangle(0.2799)$ \\
\hline III & 2.336 & ${ }^{1} E_{u}$ & 1.3035 & in-plane & $\left|H_{1} \rightarrow(L+6)\right\rangle(0.5922)$ \\
\hline & & & & & $\left|H_{2} \rightarrow(L+2)\right\rangle(0.4008)$ \\
\hline & & & & & $\left|H_{2} \rightarrow(L+5)\right\rangle(0.2602)$ \\
\hline & & & & & $\left|H_{1} \rightarrow(L+16)\right\rangle(0.2542)$ \\
\hline & 2.336 & ${ }^{1} E_{u}$ & 1.3035 & in-plane & $\left|H_{2} \rightarrow(L+6)\right\rangle(0.5922)$ \\
\hline & & & & & $\left|H_{1} \rightarrow(L+2)\right\rangle(0.4008)$ \\
\hline & & & & & $\left|H_{1} \rightarrow(L+5)\right\rangle(0.2602)$ \\
\hline & & & & & $\left|H_{2} \rightarrow(L+16)\right\rangle(0.2542)$ \\
\hline IV & 2.71 & ${ }^{1} A_{2 u}$ & 0.7969 & perpendicular & $\left|H_{2} \rightarrow(L+7) 1\right\rangle(0.5801)$ \\
\hline & & & & & $\left|H_{1} \rightarrow(L+7) 2\right\rangle(0.5801)$ \\
\hline & & & & & $|H \rightarrow 1 \rightarrow L\rangle(0.2014)$ \\
\hline & & & & & $\left|H_{1} \rightarrow L ; H_{2} \rightarrow(L+6)\right\rangle(0.1636)$ \\
\hline
\end{tabular}

